\title{
Well-posedness of non-isentropic Euler equations with physical vacuum
}

\author{
Yongcai Geng \\ Department of Mathematics, Shanghai Institute of Technology, Shanghai 201418, P. R. China. \\ yongcaigeng@alumni.sjtu.edu.cn. \\ Yachun Li \\ School of Mathematical Sciences, MOE-LSC, and SHL-MAC, \\ Shanghai Jiao Tong University, Shanghai 200240, P. R. China. \\ ycli@sjtu.edu.cn \\ Dehua Wang \\ Department of Mathematics, University of Pittsburgh, Pittsburgh, PA 15260, U.S.A. \\ dwang@math.pitt.edu. \\ Runzhang Xu \\ College of Mathematical Sciences, Harbin Engineering University, Harbin 150001, P. R. China. \\ xurunzh@hrbeu.edu.cn.
}

\begin{abstract}
We consider the local well-posedness of the one-dimensional non-isentropic compressible Euler equations with moving physical vacuum boundary condition. The physical vacuum singularity requires the sound speed to be scaled as the square root of the distance to the vacuum boundary. The main difficulty lies in the fact that the system of hyperbolic conservation laws becomes characteristic and degenerate at the vacuum boundary. Our proof is based on an approximation of the Euler equations by a degenerate parabolic regularization obtained from a specific choice of a degenerate artificial viscosity term. Then we construct the solutions to this degenerate parabolic problem and establish the estimates that are uniform with respect to the artificial viscosity parameter. Solutions to the compressible Euler equations are obtained as the limit of the vanishing artificial viscosity. Different from the isentropic case [7, 12], our momentum equation of conservation laws has an extra term $p_{S} S_{\eta}$ that leads to some extra terms in the energy function and causes more difficulties even for the case of $\gamma=2$. Moreover, we deal with this free boundary problem starting from the general cases of $2 \leq \gamma<3$ and $1<\gamma<2$ instead of only emphasizing the isentropic case of $\gamma=2$ in [7, 12, 16].
\end{abstract}

2010 Mathematics Subject Classification: Primary 35B40, 35A05, 76Y05; Secondary 35B35, 35L65.

Keywords: Non-isentropic Euler equations, vacuum boundary, free boundary problem, local well-posedness. 


\section{Introduction}

We are concerned with the one-dimensional compressible flow moving inside a dynamic vacuum boundary governed by the following non-isentropic Euler equations with initial and free boundary conditions:

$$
\begin{cases}\rho_{t}+(\rho u)_{\eta}=0, & \text { in } I(t), \\ (\rho u)_{t}+\left(\rho u^{2}+p\right)_{\eta}=0, & \text { in } I(t), \\ S_{t}+u S_{\eta}=0, & \text { in } I(t), \\ \left.(\rho, u, S)\right|_{t=0}=\left(\rho_{0}(\eta), u_{0}(\eta), S_{0}(\eta)\right), & \text { on } I(0), \\ \rho=0, & \text { on } \Gamma(t),\end{cases}
$$

where $t>0$ is the time variable, $\eta$ is the space variable; the open bounded interval $I(t) \subset \mathbf{R}$ denotes the evolving domain occupied by the gas, and $I(0)=I=\{\eta \in \mathbf{R}: 0<\eta<1\}$ is the initial spatial domain; $\Gamma(t):=\partial I(t)$ denotes the vacuum boundary that moves with the fluid velocity; $u$ represents the Eulerian velocity, and $\rho, S$ stand for the density and the entropy of the gas, respectively; $p=p(\rho, S)$ is the pressure satisfying the equation of state

$$
p=C_{\gamma} \rho^{\gamma} e^{S}, \quad 1<\gamma<3,
$$

where $C_{\gamma}$ is the adiabatic constant that is set to 1 in this paper, and $\gamma$ is the adiabatic gas exponent. The density $\rho$ satisfies the following conditions:

$$
\rho(\eta, t)>0 \text { in } \mathrm{I}(\mathrm{t}) \text { and } \rho(\eta, t)=0 \text { on } \Gamma(t) .
$$

The equations $(1.1)_{1}-(1.1)_{3}$ are the conservation laws of mass, momentum, and energy, respectively; $(1.1)_{4}$ is the initial condition for the density, velocity, and entropy; and the boundary condition (1.11) 5 states that the density must vanish along the vacuum boundary.

To understand the behavior of a solution near the vacuum $\rho=0$, similarly to [27] we take

$$
c^{2}=p_{\rho}(\rho, S)=\gamma \rho^{\gamma-1} e^{S},
$$

and rewrite $(1.1)_{1}-(1.1)_{2}$ in terms of $u$ and $c$ as

$$
\left\{\begin{array}{l}
\left(c^{2}\right)_{t}+(\gamma-1) c^{2} u_{\eta} e^{-S}+u\left(c^{2}\right)_{\eta}=0 \\
u_{t}+u u_{\eta}+\frac{1}{\gamma-1}\left(c^{2}\right)_{\eta}+\frac{c^{2}}{\gamma} S_{\eta}=0 .
\end{array}\right.
$$

The trajectory of the free boundary

$$
\Gamma(\eta, t)=c l\{(\eta, t): \rho(\eta, t)>0\} \cap c l\{(\eta, t): \rho(\eta, t)=0\}
$$

coincides with the gas particle path

$$
\frac{d \eta(t)}{d t}=u(\eta(t), t)
$$


Thus, $(1.4)_{2}$ on $\Gamma(\eta, t)$ becomes

$$
\frac{d u}{d t}=-\frac{1}{\gamma-1}\left(c^{2}\right)_{\eta}
$$

Generally, the acceleration $d u / d t$ of $\Gamma(\eta, t)$ would be finite, hence we have

$$
0<\left|\frac{\partial c^{2}(\Gamma(\eta, t), t)}{\partial \eta}\right|<\infty, \quad \text { on } \Gamma(\eta, t),
$$

which defines a physical vacuum boundary condition (or singularity). Since $\rho_{0}>0$ in $I$, this condition implies that for some positive constant $C$ and $\eta \in I$ near the vacuum boundary $\Gamma=\partial I$,

$$
\rho_{0}^{\gamma-1} \geq C \operatorname{dist}(\eta, \Gamma)
$$

Equivalently, the physical vacuum condition (1.5) implies that for some $\kappa>0$,

$$
0<C_{\kappa} \leq\left|\frac{\partial \rho_{0}^{\gamma-1}(\eta)}{\partial \eta}\right|<\infty, \quad \text { if } \operatorname{dist}(\eta, \partial I) \leq \kappa,
$$

and

$$
\rho_{0}^{\gamma-1}(\eta) \geq C_{\kappa}>0, \quad \text { if } \operatorname{dist}(\eta, \partial I) \geq \kappa,
$$

for a constant $C_{\kappa}$ depending on $\kappa$.

The mathematical analysis of vacuum states dates back to Lin [24] and Liu-Smoller [28] for the isentropic gas dynamics. The definition of physical vacuum was motivated by the study of the Euler equations with damping in [27, 30], and we refer the reader to [13, 14, 25, 27, 31, 36] for more discussions. At the vacuum boundary, the hyperbolic system of Euler equations becomes degenerate and the characteristic speeds are singular [30], then the classical theory of hyperbolic systems does not apply, thus even the local existence of smooth solutions with the physical vacuum boundary is still largely open. When the data are compactly supported, there are three ways to study the problem. The first approach consists of solving the compressible Euler system in the whole space and it requires that the system $(1.1)_{1}-(1.1)_{2}$ holds in the sense of distribution for all $x \in \mathbf{R}^{d}$ and $t \in[0, T]$, which is the strategy to construct global weak solutions in [3, 9, 26]. The second consists of symmetrizing the system first and then obtaining the local existence by the theory of symmetric hyperbolic system in the whole space (see for instances [21,34,35] for classical systems and [11,23] for relativistic systems).

This paper is concerned with the the third one which consists of requiring the Euler equations to hold on the set $\{(x, t): \rho(x, t)>0\}$ and writing an equation for the vacuum boundary $\Gamma$ that is a free boundary. Here the vacuum boundary is part of the unknowns. In this case, an appropriate boundary condition at vacuum is necessary. Suppose that the origin is the initial vacuum contact point and the sound speed $c$ behaves like $c \sim|x|^{h}$. If $h \geq 1$, the initial contact with vacuum is sufficiently smooth, the local solution to the Euler equations was obtained in [29]; if $0<h<1$, the initial contact with vacuum is Hölder continuous, the physical vacuum corresponds to $h=\frac{1}{2}$ (c.f. [4, 7, 8, 16]), and the boundary behavior seems ill-posed [17] when $0<h<\frac{1}{2}$ and $\frac{1}{2}<h<1$; see [17, 18] for more 
discussions. The case $h=0$ occurs in the regime of no continuous initial contact with vacuum. In this case, a Cauchy problem can be considered, for example, the Riemann problem was studied for the genuinely discontinuous initial data in [2,10]; and a free boundary problem can also be studied, such as [24] for the positive density at the vacuum boundary. In a series of papers [16-20] JangMasmoudi gave a rigorous and detailed proof to the existence theory with physical vacuum boundary. For the one-dimensional isentropic compressible gas with physical vacuum boundary condition, to overcome the degeneracy difficulty of propagation speed, in [16] they proposed a new formulation such that some energy estimates can be closed in the appropriate space, and moreover, they proved that the vacuum boundary behavior is preserved on some short time interval. They also investigated the multi-dimensional compressible gas flow with vacuum boundary [18]. In [19], results on free boundary problems were reviewed and some related open problems were proposed. Meanwhile, they extended their research to the compressible Navier-Stokes-Poisson system of spherically symmetric isentropic flows, and established the local-in-time well-posedness of strong solutions to the vacuum free boundary problem [20]. Countand-Shkoller [7, 8] also did many works on this free boundary problem. In [7], they adopted a different approach from Jiang-Masmoudi [16] viewing the initial density function $\rho_{0}$ as a parameter, thus the isentropic compressible system becomes a momentum conservation equation, then they used the vanishing viscosity method to establish the local existence to the isentropic one-dimensional compressible flow with physical vacuum. Recently, they also established the a priori estimates for the free boundary problem of the three-dimensional compressible Euler equations [8]. This technique proposed by Countand-Shkoller in [7] has been applied to many other systems of degenerate and characteristic hyperbolic systems of conservation laws. For example, Luo-Xin-Zeng [32] and Gu-Lei [12] recently extended this method to the spherically symmetric system and one-dimensional compressible Euler-Poisson equations with moving physical vacuum boundary. Besides the local existence theory of physical vacuum states, there are also some works on the long time behavior. Based on self-similar behavior, via Darcy's law, Liu conjectured [27] that solutions of Euler equations with damping should behave asymptotically like the solutions of the porous media equation. This problem was studied by Huang, Marcati and Pan [15] in the framework of entropy solutions by the method of compensated compactness in $L^{\infty}$, and later by Luo and Zeng [33] by tracking the vacuum boundary.

In this paper, we deal with the non-isentropic compressible Euler equations with physical vacuum. Compared with the results already obtained in [7, 12, 16], there are three novel features. The first new point is that our momentum equation has an extra term $p_{S} S_{\eta}$, because the pressure function depends on not only the density function $\rho(\eta, t)$ but also the entropy function $S(\eta, t)$ that is equal to $S_{0}(\eta)$, here $S_{0}(\eta)$ stands for the initial entropy function (in Lagrangian coordinate, the entropy function satisfies $S_{t}=0$ ). To overcome this difficulty, in the energy function we will introduce more terms like $\sum_{j=1}^{2}\left\|\omega^{2+\mu} \partial_{t}^{4-2 j} \partial_{x}^{j+2} v(\cdot, t)\right\|_{0}^{2},\left\|\omega^{1 / 2+\mu} \partial_{t} v^{\prime}(\cdot, t)\right\|_{0}^{2}$ and add a new artificial viscous term $\epsilon\left(\omega^{2+2 \mu} v^{\prime} e^{S_{0}}\right)^{\prime}$, with ${ }^{\prime}=\frac{\partial}{\partial x}$, compared with the isentropic case. To close the energy estimates, besides the similar estimates to the isentropic case, we also need to deal with these additional terms 
and the term $\epsilon\left(\omega^{2+2 \mu} e^{S_{0}}\right)^{\prime} v^{\prime}$ from the artificial viscous term. In fact, even for the case of $\gamma=2$, the energy function also has more terms than the isentropic case.

The second new feature is that we will treat general $\gamma(2 \leq \gamma<3$ and $1<\gamma<2)$ from the beginning instead of only emphasizing the isentropic case $\gamma=2$ as in [7, 12, 16]. Thus, we will face some new mathematical difficulties. For example, when $2 \leq \gamma<3$, we will deal with $\left.\left\|\frac{1}{\omega^{1 / 2-\mu}}\right\|\right|_{L^{\beta}}$ (c.f (4.29) ) instead of $\left\|\frac{1}{\rho_{0}^{1 / 2}}\right\|_{L^{\beta^{\prime}}}$ (c.f. (6.33) in [7] and (6.45) in [12]) for the case of $\gamma=2$ in the process of energy estimates, where determining the proper value for $\beta$ is technically more difficult than that for $\beta^{\prime}$ due to $-\frac{1}{4}<\mu \leq 0$.

The third new feature comes from the case $1<\gamma<2$, since the momentum equation can be written as the following equation with the distance function $\omega=\rho_{0}^{\gamma-1}$ as a parameter:

$$
\omega^{\frac{1}{\gamma-1}} v_{t}+\left(\frac{\rho_{0}^{\gamma} e^{S_{0}}}{\left(\eta^{\prime}\right)^{\gamma}}\right)_{x}=0
$$

thus we know that the coefficient of $v_{t}$ will degenerate fast as $\gamma \rightarrow 1$ near the vacuum boundary. To obtain the $H^{2}$ norm of $v$ (and thus the $C^{1}$ norm of $v$ ) for small $\gamma-1$, from the Hardy type embedding inequality (2.13), the higher energy function $\tilde{E}(t)$ defined in (2.15) for $1<\gamma<2$ implies that

$$
\|v\|_{2}^{2} \leq\|v\|_{\frac{l+1}{2}-\left(\frac{1}{2}+\mu\right)}^{2} \leq C \sum_{i=0}^{\frac{l+1}{2}}\left\|\omega^{1 / 2+\mu} \partial_{x}^{i} v\right\|_{0}^{2} \leq C \tilde{E}, \quad l=3+2\left\lceil\frac{1}{2}+\frac{1}{\gamma-1}\right\rceil,
$$

indicating the order of derivatives $l \rightarrow \infty$ as $\gamma \rightarrow 1$. For example, different from the case of $2<\gamma<3$, the estimates of higher order spatial derivatives for the case $\gamma=\frac{3}{2}$ are much more difficult, for which we use (4.31) to close the energy estimates and the details can be found in (5.15)-(5.20). Finally, we also present the proof of uniqueness for the general case $1<\gamma<3$.

The rest of the paper is organized as follows. In Section 2, we introduce the Lagrangian coordinates to transform the free boundary problem to a fixed boundary problem, and we provide some useful inequalities including the Sobolev embedding inequalities and state our main result. In Section 3 , we first present a degenerate parabolic approximation with viscosity $\varepsilon$ to the compressible Euler equations, then use a fixed point theorem to solve this approximate problem. In Section 4 and Section 5, we will prove some uniform estimates independent of $\varepsilon$ for $2 \leq \gamma<3$ and $1<\gamma<2$ respectively. Then we take the limit as $\varepsilon \rightarrow 0$ to obtain the solution of the compressible Euler equations and hence establish the local existence theorem. In Section 6, we will prove the main result, i.e., Theorem 2.1.

\section{Preliminaries and main result}

In this section, we provide some preliminaries and state the main result. 


\subsection{Lagrangian formulation}

System (1.1) is in the Eulerian coordinates $(\eta, t)$. We first rewrite it in the Lagrangian variables $(x, t)$. Let $\eta(x, t)$ denote the position of the gas particle $x$ at time $t$, then

$$
\partial_{t} \eta=u(\eta(x, t), t) \text { for } t>0 \text { and } \eta(x, 0)=x .
$$

Set the Lagrangian velocity, Lagrangian density and entropy in the following:

$$
v(x, t)=u(\eta(x, t), t), \quad f(x, t)=\rho(\eta(x, t), t), \quad \tilde{S}(x, t)=S(\eta(x, t), t) .
$$

Then

$$
\begin{gathered}
v_{t}=u_{\eta} \eta_{t}+u_{t}=u_{\eta} u+u_{t}, \quad v_{x}=u_{\eta} \eta_{x}, \\
f_{t}=\rho_{\eta} \eta_{t}+\rho_{t}=\rho_{\eta} u+\rho_{t}, \quad f_{x}=\rho_{\eta} \eta_{x}, \\
\tilde{S}_{t}=S_{\eta} \eta_{t}+S_{t}=S_{\eta} u+S_{t}, \quad \tilde{S}_{x}=S_{\eta} \eta_{x} .
\end{gathered}
$$

Using (2.1), the Lagrangian version of equations $(1.1)_{1}-(1.1)_{3}$ can be written on the fixed reference domain $I$ as

$$
\left\{\begin{array}{l}
f_{t}+f v_{x} / \eta_{x}=0 \\
f v_{t}+\left(f^{\gamma} \exp \tilde{S}\right)_{x} / \eta_{x}=0 \\
\tilde{S}_{t}=0 \\
\left.(f, v, \tilde{S}, \eta)\right|_{t=0}=\left(\rho_{0}, u_{0}, S_{0}, x\right)
\end{array}\right.
$$

From $(2.2)_{3}$ we have

$$
\tilde{S}(x, t) \equiv S_{0}(x)
$$

then (2.2) is reduced to

$$
\left\{\begin{array}{l}
f_{t}+f v_{x} / \eta_{x}=0, \\
f v_{t}+\left(f^{\gamma} e^{S_{0}}\right)_{x} / \eta_{x}=0, \\
\left.(f, v, \eta)\right|_{t=0}=\left(\rho_{0}, u_{0}, x\right) .
\end{array}\right.
$$

From $(2.4)_{1}$, we know that

$$
\left(f \eta_{x}\right)_{t}=f_{t} \eta_{x}+f \eta_{x t}=f_{t} \eta_{x}+f v_{x}=0
$$

which implies

$$
f=\rho(\eta(x, t), t)=\rho_{0} / \eta_{x}
$$

Hence, the initial density function $\rho_{0}$ can be viewed as a parameter in the Euler equations. Thus, problem (2.4) can be rewritten as

$$
\begin{cases}\rho_{0} v_{t}+\left(\frac{\rho_{0}^{\gamma} e^{S_{0}}}{\eta_{x}^{\gamma}}\right)_{x}=0, & \text { in } I \times(0, T], \\ (\eta, v)=\left(x, u_{0}\right), & \text { in } I \times\{t=0\} \\ \rho_{0}^{\gamma-1}=0, & \text { on } \Gamma,\end{cases}
$$


with $\rho_{0}^{\gamma-1} \geq C \operatorname{dist}(x, \Gamma)$ for $x$ near $\Gamma$. In the following, we adopt the notation

$$
\omega=\rho_{0}^{\gamma-1}
$$

It is obvious that $\omega=\rho_{0}$ for $\gamma=2$.

As noted above, the initial domain $I \subset \mathbf{R}$ at $t=0$ is

$$
I=(0,1)
$$

and the initial boundary points are $\Gamma=\partial I=\{0,1\}$.

\subsection{Embedding and interpolation inequalities}

For integers $k \geq 0$, let the Sobolev space $H^{k}(I)$ be the completion of $C^{\infty}(I)$ under the norm

$$
\|u\|_{k}:=\left(\sum_{a \leq k} \int_{I}\left|D^{a} u(x)\right|^{2} d x\right)^{\frac{1}{2}} .
$$

For a real number $s \geq 0$, the Sobolev spaces $H^{s}(I)$ and the norms $\|\cdot\|_{s}$ are defined by interpolation. We use $H_{0}^{1}(I)$ to denote the subspace of $H^{1}(I)$ consisting of those functions $u(x)$ vanishing at $x=0$ and $x=1$.

We denote by $\|\cdot\|_{0}$ the $L^{2}$ norm. We first review some useful embedding and interpolation inequalities. For Sobolev spaces, one has

$$
\|u\|_{L^{r}} \leq C\|u\|_{\frac{1}{2}}, \quad 1<r<+\infty
$$

We will also use the interpolation inequality [1]:

$$
\|u\|_{s} \leq C\|u\|_{s_{0}}^{1-\theta}\|u\|_{s_{1}}^{\theta}
$$

with $0 \leq s_{0} \leq s \leq s_{1}$ and $s=(1-\theta) s_{0}+\theta s_{1}(0 \leq \theta \leq 1)$. In particular, some useful inequalities in this paper are

$$
\|u\|_{\frac{3}{4}} \leq C\|u\|_{\frac{1}{2}}^{\frac{1}{2}}\|u\|_{1}^{\frac{1}{2}}, \quad\|u\|_{\frac{1}{2}} \leq C\|u\|_{0}^{\frac{1}{2}}\|u\|_{1}^{\frac{1}{2}}
$$

For simplicity, we denote by $\|\cdot\|_{\infty}$ the $L^{\infty}$ norm, then

$$
\|u\|_{\infty} \leq C_{p}\|u\|_{1}
$$

Using (2.10), one has

$$
\|u\|_{\infty} \leq C_{p}\|u\|_{\frac{3}{4}} \leq C\|u\|_{1 / 2}^{1 / 2}\|u\|_{1}^{1 / 2} \leq C\|u\|_{0}^{1 / 4}\|u\|_{1}^{3 / 4}
$$


Let $\omega$ denote the distance function to the boundary $\Gamma$. For any $a>0$ and nonnegative integer $b$, the weighted Sobolev space $H^{a, b}(I)$ is given by

$$
H^{a, b}(I):=\left\{\omega^{a / 2} F \in L^{2}(I): \int_{I} \omega^{a}(x)\left|D^{k} F(x)\right|^{2} d x<+\infty, 0 \leq k \leq b\right\}
$$

with the norm

$$
\|F\|^{a, b}(I):=\sum_{k=0}^{b} \int_{I} \omega^{a}(x)\left|D^{k} F(x)\right|^{2} d x .
$$

Then for any $b>\frac{a}{2}$, one has the following embedding [22]:

$$
H^{a, b}(I) \hookrightarrow H^{b-a / 2}(I) .
$$

For the estimates on the higher order spatial derivatives of $v$, we introduce the following lemma. Lemma 2.1 (Lemma 1 in [5]). Let $\varepsilon>0$ and $g \in L^{\infty}\left(0, T ; H^{s}(I)\right)$ be given, and let $f \in$ $H^{1}\left(0, T ; H^{s}(I)\right)$ be such that

$$
f+\frac{\varepsilon}{\gamma} f_{t}=g \quad \text { in }(0, T) \times I
$$

Then,

$$
\|f\|_{L^{\infty}\left(0, T ; H^{s}(I)\right)} \leq C \max \left\{\|f(0)\|_{s},\|g\|_{L^{\infty}\left(0, T ; H^{s}(I)\right)}\right\} .
$$

\subsection{Higher-order energy functions and main result}

To close the energy estimates and state the main theorem, we define the energy functions for the two cases of $2 \leq \gamma<3$ and $1<\gamma<2$, respectively. We consider the following higher-order energy functions:

Case I: $2 \leq \gamma<3$.

$$
\begin{aligned}
\hat{E}(t)= & \left\|\omega^{1+\mu} \partial_{t}^{4} v^{\prime}(\cdot, t)\right\|_{0}^{2}+\left\|\omega^{1+\mu} \partial_{t}^{4} v(\cdot, t)\right\|_{0}^{2} \\
& +\sum_{j=1}^{2}\left\{\left\|\omega^{3 / 2+\mu} \partial_{t}^{5-2 j} \partial_{x}^{j+1} v(\cdot, t)\right\|_{0}^{2}+\sum_{i=1}^{j}\left\|\omega^{1 / 2+\mu} \partial_{t}^{5-2 j} \partial_{x}^{i} v(\cdot, t)\right\|_{0}^{2}\right\} \\
& +\sum_{j=1}^{2}\left\{\left\|\omega^{2+\mu} \partial_{t}^{4-2 j} \partial_{x}^{j+2} v(\cdot, t)\right\|_{0}^{2}+\sum_{i=-1}^{j}\left\|\omega^{1+\mu} \partial_{t}^{4-2 j} \partial_{x}^{i+1} v(\cdot, t)\right\|_{0}^{2}\right\}
\end{aligned}
$$

where $-\frac{1}{4}<\mu=\frac{2-\gamma}{2(\gamma-1)} \leq 0$.

Case II: $1<\gamma<2$.

$$
\begin{aligned}
\tilde{E}(t)= & \left\|\omega^{1+\mu} \partial_{t}^{l} v^{\prime}(\cdot, t)\right\|_{0}^{2}+\left\|\omega^{1+\mu} \partial_{t}^{l} v(\cdot, t)\right\|_{0}^{2} \\
& +\sum_{j=1}^{\frac{l+1}{2}}\left\{\left\|\omega^{3 / 2+\mu} \partial_{t}^{l+1-2 j} \partial_{x}^{j+1} v(\cdot, t)\right\|_{0}^{2}+\sum_{i=1}^{j}\left\|\omega^{1 / 2+\mu} \partial_{t}^{l+1-2 j} \partial_{x}^{i} v(\cdot, t)\right\|_{0}^{2}\right\} \\
& +\sum_{j=1}^{\frac{l-1}{2}}\left\{\left\|\omega^{2+\mu} \partial_{t}^{l-2 j} \partial_{x}^{j+2} v(\cdot, t)\right\|_{0}^{2}+\sum_{i=-1}^{j}\left\|\omega^{1+\mu} \partial_{t}^{l-2 j} \partial_{x}^{i+1} v(\cdot, t)\right\|_{0}^{2}\right\}
\end{aligned}
$$


where

$$
\mu=\frac{2-\gamma}{2(\gamma-1)} \geq 0, \quad l=3+2\lceil 1 / 2+\mu\rceil
$$

and $\lceil\cdot\rceil$ is the following ceiling function of $q \geq 0$ :

$$
\lceil q\rceil:=\min \{m: m \geq q, m \text { is an integer }\} .
$$

Remark 2.1. From the definitions of $\mu, l$ in the case II, we find that $\gamma \rightarrow 1$ implies $l \rightarrow \infty$. Thus, for $1<\gamma<2$, the higher-order derivatives will be needed, which will cause technical difficulties compared with the case of $2 \leq \gamma<3$.

Remark 2.2. Even when $\gamma=2$, the higher-order energy function in Case $\mathbf{I}$ is different from the isentropic case in [7] 12], and the former contains the latter. In the non-isentropic case, some additional terms like $\sum_{j=1}^{2}\left\|\rho_{0}^{2} \partial_{t}^{4-2 j} \partial_{x}^{j+2} v(\cdot, t)\right\|_{0}^{2},\left\|\rho_{0}^{1 / 2} \partial_{t} v^{\prime}\right\|_{0}^{2}$ will appear due to the additional term $p_{S} S_{\eta}$ even for $\gamma=2$.

In fact, when $\gamma=2$, the energy function for the isentropic case in [7, 12] is

$$
\begin{aligned}
E_{v}(t)= & \sum_{s=0}^{4}\left\|\partial_{t}^{s} v(\cdot, t)\right\|_{2-s / 2}^{2}+\sum_{s=0}^{2}\left\|\rho_{0} \partial_{t}^{2 s} v(\cdot, t)\right\|_{3-s}^{2} \\
& +\left\|\rho_{0}^{3 / 2} \partial_{t} \partial_{x}^{3} v(\cdot, t)\right\|_{0}^{2}+\left\|\rho_{0}^{3 / 2} \partial_{t}^{3} \partial_{x}^{2} v(\cdot, t)\right\|_{0}^{2} \\
& +\left\|\rho_{0}^{1 / 2} \partial_{t} \partial_{x}^{2} v(\cdot, t)\right\|_{0}^{2}+\left\|\rho_{0}^{1 / 2} \partial_{t}^{3} \partial_{x} v(\cdot, t)\right\|_{0}^{2}
\end{aligned}
$$

Since $\gamma=2$ implies $\mu=0$ and $\omega=\rho_{0}$, the energy function in Case $\mathbf{I}$ is

$$
\begin{aligned}
\hat{E}(t)= & \left\|\rho_{0} \partial_{t}^{4} v^{\prime}(\cdot, t)\right\|_{0}^{2}+\left\|\rho_{0} \partial_{t}^{4} v(\cdot, t)\right\|_{0}^{2} \\
& +\sum_{j=1}^{2}\left\{\left\|\rho_{0}^{3 / 2} \partial_{t}^{5-2 j} \partial_{x}^{j+1} v(\cdot, t)\right\|_{0}^{2}+\sum_{i=1}^{j}\left\|\rho_{0}^{1 / 2} \partial_{t}^{5-2 j} \partial_{x}^{i} v(\cdot, t)\right\|_{0}^{2}\right\} \\
& +\sum_{j=1}^{2}\left\{\left\|\rho_{0}^{2} \partial_{t}^{4-2 j} \partial_{x}^{j+2} v(\cdot, t)\right\|_{0}^{2}+\sum_{i=-1}^{j}\left\|\rho_{0} \partial_{t}^{4-2 j} \partial_{x}^{i+1} v(\cdot, t)\right\|_{0}^{2}\right\} .
\end{aligned}
$$

From the weighted Sobolev embedding inequality (2.13), we know that

$$
\begin{gathered}
\left\|\rho_{0} \partial_{t}^{4} v^{\prime}\right\|_{0}^{2} \geq\left\|\partial_{t}^{4} v\right\|_{1-1}^{2}=\left\|\partial_{t}^{4} v\right\|_{0}^{2} \\
\sum_{j=1}^{2}\left\|\rho_{0}^{2} \partial_{t}^{4-2 j} \partial_{x}^{j+2} v(\cdot, t)\right\|_{0}^{2} \geq \sum_{j=1}^{2}\left\|\partial_{t}^{4-2 j} v(\cdot, t)\right\|_{j+2-2}^{2}=\sum_{j=1}^{2}\left\|\partial_{t}^{4-2 j} v(\cdot, t)\right\| \|_{j}^{2},
\end{gathered}
$$

and

$$
\sum_{j=1}^{2}\left\|\rho_{0}^{3 / 2} \partial_{t}^{5-2 j} \partial_{x}^{j+1} v(\cdot, t)\right\|_{0}^{2} \geq \sum_{j=1}^{2}\left\|\partial_{t}^{5-2 j} v(\cdot, t)\right\|_{j-1 / 2}^{2}
$$


Thus, we have

$$
\sum_{s=0}^{4}\left\|\partial_{t}^{s} v(\cdot, t)\right\|_{2-s / 2}^{2} \leq \hat{E}(t)
$$

When $j=1,2$, the first term on the second line in (2.17) deduces to the second line of 2.16). When $j=2, i=2,3$, the second term in the second line in (2.17) leads to the third line of 2.16). Comparing (2.16) with (2.17), the latter has some additional terms $\sum_{j=1}^{2}\left\|\rho_{0}^{2} \partial_{t}^{4-2 j} \partial_{x}^{j+2} v(\cdot, t)\right\|_{0}^{2}$, $\| \omega^{1 / 2+\mu} \partial_{t} v^{\prime}||_{0}^{2}$ caused by $p_{S} S_{\eta}$ in the non-isentropic case. The detailed analysis can be seen from the higher-order spatial derivative estimates in Subsection 4.2.

For simplicity, we introduce

$$
E(t)= \begin{cases}\hat{E}(t), & 2 \leq \gamma<3 \\ \tilde{E}(t), & 1<\gamma<2\end{cases}
$$

We now state our main result as follows.

Theorem 2.1. Given the initial data $\left(\rho_{0}, u_{0}, S_{0}\right)$ with $E(0)<\infty, \rho_{0}(x)>0$ in $I$, if the physical vacuum condition (1.6) holds for $\rho_{0}$, and

$$
\underline{S} \leq S_{0}^{\prime}(x) \leq \bar{S}
$$

in I for some positive constants $\underline{S}$ and $\bar{S}$, there exists a unique solution to (2.5) (and hence (1.1)) on $[0, T]$ for some $T>0$ sufficiently small, such that,

$$
\sup _{t \in[0, T]} E(t) \leq P(E(0))
$$

where $P$ is some polynomial function of its argument.

We note that we shall use $P(\cdot)$ to denote a generic polynomial function of its argument, and $P$ will change from line to line with no explicit expressions necessarily given in the paper.

\section{The degenerate parabolic approximation of the system}

For convenience, we write ${ }^{\prime}=\frac{\partial}{\partial x}$. Now for $\varepsilon>0$, we consider the following nonlinear degenerate parabolic approximation of (2.5):

$$
\rho_{0} v_{t}+\left(\frac{\rho_{0}^{\gamma} e^{S_{0}}}{\left(\eta^{\prime}\right)^{\gamma}}\right)^{\prime}=\varepsilon\left(\rho_{0}^{\gamma} v^{\prime} e^{S_{0}}\right)^{\prime} \quad \text { in } I \times[0, T] .
$$

With $\rho_{0}^{\gamma-1}=\omega \geq C \operatorname{dist}(x, \Gamma)$ for $x \in I$ near $\partial I$, (3.1) with the initial and boundary conditions becomes

$$
\begin{cases}\omega^{1+2 \mu} v_{t}+\left(\frac{\omega^{2+2 \mu} e^{S_{0}}}{\left(\eta^{\prime}\right)^{\gamma}}\right)^{\prime}=\varepsilon\left(\omega^{2+2 \mu} v^{\prime} e^{S_{0}}\right)^{\prime}, & \text { in } I \times[0, T], \\ (v, \eta)=\left(u_{0}, x\right), & \text { in } I \times\{t=0\} \\ \rho_{0}=0, & \text { on } \Gamma,\end{cases}
$$


where $\mu=\frac{2-\gamma}{2(\gamma-1)}$. Compared with the isentropic case [7, 12], this nonlinear equation has an additional term $\varepsilon \omega^{2+2 \mu} v^{\prime} e^{S_{0}} S_{0}^{\prime}$, which will cause more technical difficulties in the following proofs and computations.

Given $u_{0}$ and $\rho_{0}$ and using the fact that $\eta(x, 0)=x, \eta^{\prime}(x, 0)=1$ we can compute the quantity $\left.v_{t}\right|_{t=0}$ for the degenerate parabolic $\varepsilon$-problem (3.2) by using (3.1):

$$
\begin{aligned}
\left.v_{t}\right|_{t=0} & =\left.\left(-\frac{1}{\rho_{0}}\left(\left(\frac{\rho_{0}}{\eta^{\prime}}\right)^{\gamma} e^{S_{0}}\right)^{\prime}+\varepsilon \frac{1}{\rho_{0}}\left(\rho_{0}^{\gamma} v^{\prime} e^{S_{0}}\right)^{\prime}\right)\right|_{t=0} \\
& =-\omega e^{S_{0}} S_{0}^{\prime}+\frac{\gamma}{\gamma-1} \omega^{\prime}\left(\varepsilon u_{0}^{\prime}-1\right) e^{S_{0}}+\varepsilon \omega\left(u_{0}^{\prime \prime}+u_{0}^{\prime} S_{0}^{\prime}\right) e^{S_{0}} .
\end{aligned}
$$

Similarly, for all $k \in \mathbb{N}$,

$$
u_{k}:=\left.\partial_{t}^{k} v\right|_{t=0}=\left.\partial_{t}^{k-1}\left(-\frac{1}{\rho_{0}}\left(\left(\frac{\rho_{0}}{\eta^{\prime}}\right)^{\gamma} e^{S_{0}}\right)^{\prime}+\frac{\varepsilon}{\rho_{0}}\left(\rho_{0}^{\gamma} v^{\prime} e^{S_{0}}\right)^{\prime}\right)\right|_{t=0}
$$

These formulas show that each $\left.\partial_{t}^{k} v\right|_{t=0}$ is a function of spatial derivatives of $u_{0}$ and $\rho_{0}$.

As in [6, 7, 12], the linearized problem of (3.2) is

$$
\begin{cases}\omega^{1+2 \mu} v_{t}+\left(\frac{\omega^{2+2 \mu} e^{S_{0}}}{\left(\bar{\eta}^{\prime}\right)^{\gamma}}\right)^{\prime}=\varepsilon\left(\omega^{2+2 \mu} v^{\prime} e^{S_{0}}\right)^{\prime}, & \text { in } I \times[0, T], \\ (v, \eta)=\left(u_{0}, x\right), & \text { in } I \times\{t=0\} \\ \rho_{0}=0, & \text { on } \Gamma,\end{cases}
$$

where $\bar{\eta}=x+\int_{0}^{t} \bar{v}(x, \tau) d \tau$ and $\bar{v}$ is given, the existence and the uniqueness of the solution $v^{\varepsilon}$ can be obtained by the standard arguments for the above degenerate parabolic problem (3.2) in a time interval $\left[0, T^{\varepsilon}\right]$ with sufficiently smooth initial data, using the fixed point argument. Henceforth, we assume that $T^{\varepsilon}>0$ is sufficiently small such that, independent of the choice of $v^{\varepsilon}$,

$$
\eta^{\varepsilon}(x, t)=x+\int_{0}^{t} v^{\varepsilon}(x, s) d s
$$

is injective for $t \in\left[0, T^{\varepsilon}\right]$, and $\frac{1}{2} \leq \eta^{\prime}(x, t) \leq \frac{3}{2}$ for $t \in\left[0, T^{\varepsilon}\right]$ and $x \in \bar{I}$.

We will establish the a priori estimates uniform in $\varepsilon$ to show that the time of existence does not depend on $\varepsilon$, and then take the weak limit as $\varepsilon \rightarrow 0$ of the sequence of solutions to (3.2) to obtain the existence of solution to the Euler system (1.1).

\section{Uniform estimates of $v^{\varepsilon}$ for $2 \leq \gamma<3$}

Our objective in this section is to prove the uniform estimates of $v^{\varepsilon}$ for $2 \leq \gamma<3$. For the sake of notational convenience, we omit the superscript $\varepsilon$. We first give some analysis on $\hat{E}(t)$ in (2.14) 
in order to establish the desired estimates. From the weighted Sobolev embedding inequality (2.13) and $-\frac{1}{4} \leq \mu \leq 0$ as $2 \leq \gamma<3$, we have, for the first term in (2.14),

$$
\left\|\omega^{1+\mu} \partial_{t}^{4} v^{\prime}\right\|_{0}^{2} \geq\left\|\partial_{t}^{4} v\right\|_{1-1-\mu}^{2} \geq\left\|\partial_{t}^{4} v\right\|_{0}^{2}
$$

for the first term of the second line in (2.14),

$$
\sum_{j=1}^{2}\left\|\omega^{3 / 2+\mu} \partial_{t}^{5-2 j} \partial_{x}^{j+2} v(\cdot, t)\right\|_{0}^{2} \geq\|\| \partial_{t}^{5-2 j} v(\cdot, t)\left\|_{j+2-3 / 2-\mu}^{2} \geq \sum_{j=1}^{2}\right\| \partial_{t}^{5-2 j} v \|_{j-1 / 2}^{2}
$$

and for the first term of the third line in (2.14),

$$
\sum_{j=1}^{2}\left\|\omega^{2+\mu} \partial_{t}^{4-2 j} \partial_{x}^{i+2} v(\cdot, t)\right\|_{0}^{2} \geq \sum_{j=1}^{2}\left\|\partial_{t}^{4-2 j} v\right\|_{j+2-2-\mu}^{2} \geq \sum_{j=1}^{2}\left\|\partial_{t}^{4-2 j} v\right\|_{j}^{2} .
$$

Thus, (4.1)-(4.3) lead to

$$
\hat{E}(t) \geq \sum_{s=0}^{4}\left\|\partial_{t}^{s} v\right\|_{2-s / 2}^{2}
$$

\subsection{Some $\varepsilon$-independent energy estimates on the $\partial_{t}^{k}$-problem}

Proposition 4.1. For $2 \leq \gamma<3$, there exists a constant $\alpha \in(0,1)$, such that one has the following $\varepsilon$-independent energy estimate for $\partial_{t}^{5}$ - problem of $(3.2)_{1}$ :

$$
\begin{aligned}
&\left\|\omega^{1 / 2+\mu} \partial_{t}^{5} v\right\|_{0}^{2}+\left\|\omega^{1+\mu} \partial_{t}^{4} v^{\prime}\right\|_{0}^{2}+\left\|\omega^{1+\mu} \partial_{t}^{4} v\right\|_{0}^{2}+\varepsilon \int_{0}^{t}\left\|\omega^{1+\mu} \partial_{t}^{5} v^{\prime}\right\|_{0}^{2} \\
& \leq \hat{E}^{\alpha}\left(\hat{M}_{0}+\operatorname{CtP}\left(\sup _{[0, t]} \hat{E}\right)\right)
\end{aligned}
$$

with $\hat{M}_{0}=P(\hat{E}(0))$ and $P(\cdot)$ some polynomial function.

Proof. We take the fifth partial derivative $\partial_{t}^{5}$ in (3.2) and multiply it by $\partial_{t}^{5} v$, after integrating by parts, we have the following identity:

$$
\frac{1}{2} \frac{d}{d t} \int_{I} \omega^{1+2 \mu}\left|\partial_{t}^{5} v\right|^{2}-\int_{I} \partial_{t}^{5}\left(\frac{\omega^{2+2 \mu}}{\left(\eta^{\prime}\right)^{\gamma}}\right) \partial_{t}^{5} v^{\prime} e^{S_{0}}+\varepsilon \int_{I} \omega^{2+2 \mu}\left|\partial_{t}^{5} v^{\prime}\right|^{2} e^{S_{0}}=0
$$

where $\omega=\rho_{0}^{\gamma-1},-\frac{1}{4}<\mu=\frac{2-\gamma}{2(\gamma-1)} \leq 0$. We see that the second term on the left-hand side of (4.6) 
can be written as

$$
\begin{aligned}
-\int_{I} \partial_{t}^{5}\left(\frac{\omega^{2+2 \mu}}{\left(\eta^{\prime}\right)^{\gamma}}\right) \partial_{t}^{5} v^{\prime} e^{S_{0}}= & \gamma \int_{I} \frac{\omega^{2+2 \mu}}{\left(\eta^{\prime}\right)^{\gamma+1}} \partial_{t}^{4} v^{\prime} \partial_{t}^{5} v^{\prime} e^{S_{0}} \\
& +\sum_{i=1}^{4} c_{i} \int_{I} \omega^{2+2 \mu} \partial_{t}^{i} \frac{1}{\left(\eta^{\prime}\right)^{\gamma+1}} \partial_{t}^{4-i} v^{\prime} \partial_{t}^{5} v^{\prime} e^{S_{0}} \\
= & \frac{\gamma}{2} \frac{d}{d t} \int_{I} \frac{\omega^{2+2 \mu}}{\left(\eta^{\prime}\right)^{\gamma+1}}\left|\partial_{t}^{4} v^{\prime}\right|^{2} e^{S_{0}} \\
& +\frac{(\gamma+1) \gamma}{2} \int_{I} \frac{\omega^{2+2 \mu}}{\left(\eta^{\prime}\right)^{\gamma+2}} v^{\prime}\left|\partial_{t}^{4} v^{\prime}\right|^{2} e^{S_{0}} \\
& +\sum_{i=1}^{4} c_{i} \int_{I} \partial_{t}^{i} \frac{1}{\left(\eta^{\prime}\right)^{\gamma+1}} \partial_{t}^{4-i} v^{\prime} \omega^{2+2 \mu} \partial_{t}^{5} v^{\prime} e^{S_{0}}
\end{aligned}
$$

Hence, substituting (4.7) into (4.6), integrating it from 0 to $t$, we find that

$$
\begin{aligned}
& \frac{1}{2} \int_{I} \omega^{1+2 \mu}\left|\partial_{t}^{5} v\right|^{2}+\frac{\gamma}{2} \int_{I}\left|\partial_{t}^{4} v^{\prime}\right|^{2} \frac{\omega^{2+2 \mu}}{\left(\eta^{\prime}\right)^{\gamma+1}} e^{S_{0}}+\varepsilon \int_{0}^{t} \int_{I} \omega^{2+2 \mu}\left|\partial_{t}^{5} v^{\prime}\right|^{2} e^{S_{0}} \\
& =\frac{1}{2} \int_{I} \omega^{1+2 \mu}\left|\partial_{t}^{5} v_{0}\right|^{2}+\left.\frac{\gamma}{2} \int_{I} \frac{\omega^{2+2 \mu}}{\left(\eta^{\prime}\right)^{\gamma+1}}\right|_{t=0}\left|\partial_{t}^{4} v_{0}^{\prime}\right|^{2} e^{S_{0}} \\
& \quad-\frac{(\gamma+1) \gamma}{2} \int_{0}^{t} \int_{I} \frac{\omega^{2+2 \mu}}{\left(\eta^{\prime}\right)^{\gamma+2}} v^{\prime}\left|\partial_{t}^{4} v^{\prime}\right|^{2} e^{S_{0}} \\
& \quad-\sum_{i=1}^{4} c_{i} \int_{0}^{t} \int_{I} \partial_{t}^{i} \frac{1}{\left(\eta^{\prime}\right)^{\gamma+1}} \partial_{t}^{4-i} v^{\prime} \omega^{2+2 \mu} \partial_{t}^{5} v^{\prime} e^{S_{0}} \\
& =\sum_{j=1}^{4} I_{j} .
\end{aligned}
$$

It is easy to verify that $I_{1}, I_{2}$ can be controlled by $\hat{M}_{0}$. Now we estimate $I_{3}, I_{4}$ on the right hand-side of (4.8).

$$
\begin{aligned}
I_{3} & =-\frac{(\gamma+1) \gamma}{2} \int_{0}^{t} \int_{I} \frac{\omega^{2+2 \mu}}{\left(\eta^{\prime}\right)^{\gamma+2}} v^{\prime}\left|\partial_{t}^{4} v^{\prime}\right|^{2} e^{S_{0}} \leq C \int_{0}^{t}\left\|v^{\prime}\right\|_{\infty}\left\|\omega^{1+\mu} \partial_{t}^{4} v^{\prime}\right\|_{0}^{2} \\
& \leq C \int_{0}^{t}\|v\|_{2}\left\|\omega^{1+\mu} \partial_{t}^{4} v^{\prime}\right\|_{0}^{2} \leq C t P\left(\sup _{[0, t]} \hat{E}\right),
\end{aligned}
$$

where we have used (2.20), (4.4) and $1 / 2 \leq \eta^{\prime} \leq 3 / 2$. Then, using integration by part in time, we 
have

$$
\begin{aligned}
I_{4}= & -\sum_{i=1}^{4} c_{i} \int_{0}^{t} \int_{I} \partial_{t}^{i} \frac{1}{\left(\eta^{\prime}\right)^{\gamma+1}} \partial_{t}^{4-i} v^{\prime} \omega^{2+2 \mu} \partial_{t}^{5} v^{\prime} e^{S_{0}} \\
= & \underbrace{\int_{0}^{t} \int_{I}\left(\sum_{i=1}^{4} c_{i} \partial_{t}^{i} \frac{1}{\left(\eta^{\prime}\right)^{\gamma+1}} \partial_{t}^{4-i} v^{\prime}\right)_{t} \omega^{2+2 \mu} \partial_{t}^{4} v^{\prime} e^{S_{0}}}_{J} \\
& +\left.\int_{I} \sum_{i=1}^{4} c_{i} \partial_{t}^{i} \frac{1}{\left(\eta^{\prime}\right)^{\gamma+1}} \partial_{t}^{4-i} v^{\prime} \omega^{2+2 \mu} \partial_{t}^{4} v^{\prime}\right|_{0} ^{t} e^{S_{0}} .
\end{aligned}
$$

where

$$
J:=\int_{0}^{t} \sum_{i=1}^{10} J_{i} d t, \quad \text { and } J_{i}=\int_{I} R\left(\eta^{\prime}\right) j_{i} \omega^{2+2 \mu} \partial_{t}^{4} v^{\prime} d x,
$$

the terms $j_{i}, i=1,2 \cdots, 10$ are the functions of $v^{\prime}, \partial_{t}^{k} v^{\prime}, k=1,2,3,4$, as in the following:

$$
\begin{aligned}
& j_{1}=\partial_{t}^{4} v^{\prime} v^{\prime}, \quad j_{2}=\partial_{t}^{3} v^{\prime}\left(v^{\prime}\right)^{2}, \quad j_{3}=\partial_{t}^{3} v^{\prime} \partial_{t} v^{\prime}, \quad j_{4}=\partial_{t}^{2} v^{\prime} \partial_{t} v^{\prime} v^{\prime}, \quad j_{5}=\left(\partial_{t}^{2} v^{\prime}\right)^{2}, \\
& j_{6}=\partial_{t}^{2} v^{\prime}\left(v^{\prime}\right)^{3}, \quad j_{7}=\left(\partial_{t} v^{\prime}\right)^{3}, \quad j_{8}=\left(\partial_{t} v^{\prime}\right)^{2}\left(v^{\prime}\right)^{2}, \quad j_{9}=\partial_{t} v^{\prime}\left(v^{\prime}\right)^{4}, \quad j_{10}=\left(v^{\prime}\right)^{6},
\end{aligned}
$$

where $R\left(\eta^{\prime}\right)$ denotes some power functions of $\eta^{\prime}$. We first note that

$$
\left|J_{1}\right| \leq C|| v^{\prime}\left\|_{\infty}\right\| R\left(\eta^{\prime}\right)\left\|_{\infty}\right\| \omega^{1+\mu} \partial_{t}^{4} v^{\prime} \|_{0}^{2} \leq C P\left(\sup _{[0, t]} \hat{E}\right)
$$

where we have used (2.12) and (4.4), which means that

$$
\left\|v^{\prime}\right\|_{\infty} \leq C\left\|v^{\prime}\right\|_{0}^{1 / 4}\left\|v^{\prime}\right\|_{1}^{3 / 4} \leq C \hat{E}
$$

For $J_{2}$, we have

$$
\left|J_{2}\right| \leq\left\|R\left(\eta^{\prime}\right)\right\|_{\infty}\left\|\omega ^ { 1 / 2 + \mu } \partial _ { t } ^ { 3 } v ^ { \prime } \left|\left\|_{0}|| \omega\right\|_{\infty}^{1 / 2}\left\|v^{\prime}\right\|_{\infty}^{2}\left\|\omega^{1+\mu} \partial_{t}^{4} v^{\prime}\right\|_{0} \leq C P\left(\sup _{\in[0, t]} \hat{E}\right) .\right.\right.
$$

Similarly, we have

$$
\begin{aligned}
\left|J_{3}\right| & \leq\left\|R\left(\eta^{\prime}\right)\right\|_{\infty}\left\|\omega^{1+\mu} \partial_{t}^{3} v^{\prime} \mid\right\|_{L^{4}}\left\|\partial_{t} v^{\prime}\right\|_{L^{4}}\left\|\omega^{1+\mu} \partial_{t}^{4} v^{\prime}\right\|_{0} \\
& \leq C\left\|\omega^{1+\mu} \partial_{t}^{3} v^{\prime}\right\|_{1 / 2}\left\|\partial_{t} v^{\prime}\right\|_{\frac{1}{2}}\left\|\omega^{1+\mu} \partial_{t}^{4} v^{\prime}\right\|_{0}^{2} \leq C P\left(\sup _{[0, t]} \hat{E}\right),
\end{aligned}
$$

where we have used the physical vacuum condition (1.7) and

$$
\left\|\omega^{1 / 2} \partial_{x}\left(\omega^{1+\mu} \partial_{t}^{3} v^{\prime}\right)\right\|_{0}^{2} \leq\left\|\omega^{3 / 2+\mu} \partial_{t}^{3} v^{\prime \prime}||_{0}^{2}+\right\| \omega^{1 / 2+\mu} \omega^{\prime} \partial_{t}^{3} v^{\prime} \|_{0}^{2} \leq \hat{E} .
$$

Using 2.13), we have

$$
\left\|\omega^{1+\mu} \partial_{t}^{3} v^{\prime}\right\|_{1 / 2}=\left\|\omega^{1+\mu} \partial_{t}^{3} v^{\prime}\right\|_{1-\frac{1}{2}} \leq\left\|\omega^{1 / 2} \partial_{x}\left(\omega^{1+\mu} \partial_{t}^{3} v^{\prime}\right)\right\|_{0} \leq \hat{E}
$$


and similarly,

$$
\left|J_{4}\right| \leq C|| R\left(\eta^{\prime}\right)\left\|_{\infty}|| \omega^{1+\mu} \partial_{t}^{2} v^{\prime}||_{L^{4}}\right\| \partial_{t} v^{\prime}\left\|_{L^{4}}|| v^{\prime}\right\|_{\infty}\left\|\omega^{1+\mu} \partial_{t}^{4} v^{\prime}\right\|_{0} \leq \hat{M}_{0}+C t P\left(\sup _{[0, t]} \hat{E}\right)
$$

where we have used the fact

$$
\left\|\omega^{1+\mu} \partial_{t}^{2} v^{\prime}\right\|_{1 / 2}=\left\|\omega^{1+\mu} u_{2}^{\prime}+\int_{0}^{t} \omega^{1+\mu} \partial_{t}^{3} v^{\prime}\right\|_{1 / 2}
$$

Similarly, $J_{5}$ and $J_{6}$ can be estimated as

$$
\begin{aligned}
& \left|J_{5}\right| \leq C\left\|R\left(\eta^{\prime}\right)\right\|_{\infty}\left\|\omega^{1+\mu} \partial_{t}^{2} v^{\prime}\right\|_{L^{4}}^{2}|| \omega^{1+\mu} \partial_{t}^{4} v^{\prime} \|_{0} \leq \hat{M}_{0}+C t P\left(\sup _{[0, t]} \hat{E}\right), \\
& \left|J_{6}\right| \leq C\left\|R\left(\eta^{\prime}\right)\right\|_{\infty}\left\|\omega^{1+\mu} \partial_{t}^{2} v^{\prime}\right\|_{0}\left\|v^{\prime}\right\|_{\infty}^{3}\left\|\omega^{1+\mu} \partial_{t}^{4} v^{\prime}\right\|_{0} \leq C t P\left(\sup _{[0, t]} \hat{E}\right) .
\end{aligned}
$$

For $J_{7}-J_{10}$, using (2.8) and (4.4), we have

$$
\begin{aligned}
\left|J_{7}\right| & \leq C\left\|R\left(\eta^{\prime}\right)\right\|_{\infty}\left\|\partial_{t} v^{\prime}||_{L^{6}}^{3}\right\| \omega\left\|_{\infty}^{1+\mu}\right\| \omega^{1+\mu} \partial_{t}^{4} v^{\prime}\|\|_{0} \\
& \leq C\left\|\partial_{t} v^{\prime}||_{1 / 2}^{3}\right\| \omega^{1+\mu} \partial_{t}^{4} v^{\prime} \|_{0} \leq C P\left(\sup _{[0, t]} \hat{E}\right)
\end{aligned}
$$

and

$$
\begin{aligned}
& \left|J_{8}\right| \leq C\left\|R\left(\eta^{\prime}\right)\right\|_{\infty}\left\|\partial_{t} v^{\prime}\right\|_{L^{4}}^{2}\left\|v^{\prime}\right\|_{\infty}^{2}\|\omega\|_{\infty}^{1+\mu}\left\|\omega^{1+\mu} \partial_{t}^{4} v^{\prime}\right\|_{0} \leq C P\left(\sup _{[0, t]} \hat{E}\right) \\
& \left|J_{9}\right| \leq C\left\|R\left(\eta^{\prime}\right)\right\|_{\infty}\|\omega\|_{\infty}^{1 / 2}\left\|v^{\prime}\right\|_{\infty}^{4}\left\|\omega^{1 / 2+\mu} \partial_{t} v^{\prime}\right\|\left\|_{0}\right\| \omega\left\|_{\infty}^{1+\mu}\right\| \omega^{1+\mu} \partial_{t}^{4} v^{\prime} \|_{0} \leq C P\left(\sup _{[0, t]} \hat{E}\right)
\end{aligned}
$$

and

$$
\left|J_{10}\right| \leq C|| R\left(\eta^{\prime}\right)\left\|_{\infty}\right\| v^{\prime}||_{L^{12}}^{6}|| \omega\left\|_{\infty}^{1+\mu}|| \omega^{1+\mu} \partial_{t}^{4} v^{\prime}\right\|_{0} \leq C\left\|\omega^{1+\mu} \partial_{t}^{4} v^{\prime}\right\|_{0}\left\|v^{\prime}\right\|_{1 / 2}^{6} \leq C P\left(\sup _{[0, t]} \hat{E}\right) .
$$

Next, we treat the second term on the right-hand side of (4.10), beginning with the case of $i=1$. We see that for $\delta>0$,

$$
\begin{aligned}
\left.\int_{I} \partial_{t} \frac{1}{\eta^{\prime(\gamma+1)}} \partial_{t}^{3} v^{\prime} \omega^{2+2 \mu} \partial_{t}^{4} v^{\prime}\right|_{0} ^{t}= & \int_{I}\left(R\left(\eta^{\prime}\right) \partial_{t}^{3} v^{\prime} v^{\prime} \omega^{2+2 \mu} \partial_{t}^{4} v^{\prime}\right)(t) \\
& -\int_{I}\left(R\left(\eta^{\prime}\right) \partial_{t}^{3} v^{\prime} v^{\prime} \omega^{2+2 \mu} \partial_{t}^{4} v^{\prime}\right)(0)
\end{aligned}
$$

and

$$
\begin{aligned}
\int_{I}\left(R\left(\eta^{\prime}\right) \partial_{t}^{3} v^{\prime} v^{\prime} \omega^{2+2 \mu} \partial_{t}^{4} v^{\prime}\right)(t) & \leq C\left\|v^{\prime}\right\|_{\infty}\left\|\omega^{1+\mu} \partial_{t}^{3} v^{\prime}\right\|_{0}\left\|\omega^{1+\mu} \partial_{t}^{4} v^{\prime}\right\|_{0} \\
& \leq C \hat{E}(t)^{3 / 4}\left(M_{0}+C t P\left(\sup _{[0, t]} \hat{E}\right)\right.
\end{aligned}
$$


The case when $i=2,3,4$ can be estimated in the same fashion. Using (2.19) again, there exists a constant $\alpha$, such that all the estimates (4.8)-(4.13) together yield

$$
\begin{aligned}
\left\|\omega^{1 / 2+\mu} \partial_{t}^{5} v\right\|^{2} & +\left\|\omega^{1+\mu} \partial_{t}^{4} v^{\prime}\right\|^{2}+\varepsilon \int_{0}^{t}\left\|\omega^{1+\mu} \partial_{t}^{5} v^{\prime}\right\|^{2} \\
& \leq \hat{E}^{\alpha}\left(\hat{M}_{0}+C t P\left(\sup _{[0, t]} \hat{E}\right)\right), \quad 0<\alpha<1 .
\end{aligned}
$$

Since $\partial_{t}^{l} v=u_{l}+\int_{0}^{t} \partial_{t}^{l+1} v$, we have

$$
\left\|\omega^{1+\mu} \partial_{t}^{l} v\right\|_{0}^{2} \leq \hat{M}_{0}+C t\|\omega\|_{\infty}\left\|\omega^{1 / 2+\mu} \partial_{t}^{l+1} v\right\|_{0}^{2} .
$$

Letting $l=4$ and using first term in the energy estimate (4.14), we have

$$
\left\|\omega^{1+\mu} \partial_{t}^{4} v\right\|_{0}^{2} \leq \hat{E}^{\alpha}\left(\hat{M}_{0}+C t P\left(\sup _{[0, t]} \hat{E}\right)\right) .
$$

Thus, the proof of Proposition 4.1 is complete.

Using the same argument as proving Proposition 4.1, we can consider the $\varepsilon$-independent energy estimates for the $\partial_{t}^{3}$-problem and $\partial_{t}$-problem of (3.2) and obtain the following estimates:

Proposition 4.2. For $2 \leq \gamma<3$, there exists a constant $\alpha \in(0,1)$, such that one has the following energy estimates uniform in $\varepsilon$ :

$$
\left\|\omega^{1 / 2+\mu} \partial_{t}^{3} v\right\|_{0}^{2}+\left\|\omega^{1+\mu} \partial_{t}^{2} v^{\prime}\right\|_{0}^{2}+\left\|\omega^{1+\mu} \partial_{t}^{2} v\right\|_{0}^{2}+\varepsilon \int_{0}^{t}\left\|\omega^{1+\mu} \partial_{t}^{3} v^{\prime}\right\|_{0}^{2} \leq \hat{E}^{\alpha}\left(\hat{M}_{0}+C t P\left(\sup _{[0, t]} \hat{E}\right)\right)
$$

and

$$
\left\|\omega^{1 / 2+\mu} \partial_{t} v\right\|_{0}^{2}+\left\|\omega^{1+\mu} v^{\prime}\right\|_{0}^{2}+\left\|\omega^{1+\mu} v\right\|_{0}^{2}+\varepsilon \int_{0}^{t}\left\|\omega^{1+\mu} \partial_{t} v^{\prime}\right\|_{0}^{2} \leq \hat{E}^{\alpha}\left(\hat{M}_{0}+C t P\left(\sup _{[0, t]} \hat{E}\right)\right) .
$$

\subsection{Estimates of higher-order spatial derivatives}

Having obtained the uniform energy estimates in Propositions 4.1-4.2, we can begin our estimates of higher-order spatial derivatives. We consider $\partial_{t}^{k+1}$-problem of (3.2):

$$
\left(\omega^{2+2 \mu} \partial_{t}^{k} v^{\prime} e^{S_{0}}\right)^{\prime}-\frac{\varepsilon}{\gamma} \partial_{t}\left(\omega^{2+2 \mu} \partial_{t}^{k} v^{\prime} e^{S_{0}}\right)^{\prime}=g,
$$

where

$$
\begin{aligned}
g= & -\frac{1}{\gamma} \omega^{1+2 \mu} \partial_{t}^{k+2} v+\sum_{i=1}^{k}\left(c_{i} \omega^{2+2 \mu} \partial_{t}^{i} \frac{1}{\left(\eta^{\prime}\right)^{\gamma+1}} \partial_{t}^{k-i} v^{\prime} e^{S_{0}}\right)^{\prime} \\
& +\left[\left(1-\frac{1}{\left(\eta^{\prime}\right)^{\gamma+1}}\right)\left(\omega^{2+2 \mu} \partial_{t}^{k} v^{\prime} e^{S_{0}}\right)^{\prime}\right]-(\gamma+1) \frac{\omega^{2+2 \mu} \partial_{t}^{k} v^{\prime} e^{S_{0}} \eta^{\prime \prime}}{\left(\eta^{\prime}\right)^{\gamma+2}} .
\end{aligned}
$$


Applying Lemma 2.1 directly, we have

$$
\left\|\omega^{2+2 \mu} \partial_{t}^{k} v^{\prime}\right\|_{0} \leq C \max \left\{\|g\|_{0},\|f(0)\|_{0}\right\}
$$

However, this estimate is not good enough to obtain the corresponding estimates with weights of $\omega^{1 / 2+\mu}, \omega^{3 / 2+\mu}, \omega^{1+\mu}, \omega^{2+\mu}$ in the energy function $\hat{E}(t)$. To obtain the desired estimates, we shall reduce (4.16) to some new equations of the form in Lemma 2.1 by multiplying (4.16) by some suitable multipliers.

Proposition 4.3. For $2 \leq \gamma<3$, there exists a constant $\alpha \in(0,1)$, such that one has the following estimates:

$$
\begin{aligned}
& \sup _{[0, t]}\left(\left\|\omega^{1 / 2+\mu} \partial_{t}^{3} v^{\prime}\right\|_{0}^{2}+\left\|\omega^{3 / 2+\mu} \partial_{t}^{3} v^{\prime \prime}\right\|_{0}^{2}\right) \leq C\left(\hat{E}^{\alpha}+1\right)\left(\hat{M}_{0}+C t P\left(\sup _{[0, t]} \hat{E}\right)\right)+C t P\left(\sup _{[0, t]} \hat{E}\right), \\
& \sup _{[0, t]}\left(\left\|\omega^{1 / 2+\mu} \partial_{t} v^{\prime \prime}\right\|_{0}^{2}+\left\|\omega^{3 / 2+\mu} \partial_{t} v^{\prime \prime \prime}\right\|_{0}^{2}\right) \leq C\left(\hat{E}^{\alpha}+1\right)\left(\hat{M}_{0}+C t P\left(\sup _{[0, t]} \hat{E}\right)\right)+C t P\left(\sup _{[0, t]} \hat{E}\right) .
\end{aligned}
$$

Proof. First, choosing $k=3$ in (4.16), and multiplying both sides of (4.16) by $\omega^{-(1 / 2+\mu)}$, we have

$$
\begin{aligned}
\omega^{-(1 / 2+\mu)}\left(\omega^{2+2 \mu} \partial_{t}^{3} v^{\prime} e^{S_{0}}\right)^{\prime} & -\frac{\varepsilon}{\gamma} \partial_{t}\left[\omega^{-(1 / 2+\mu)}\left(\omega^{2+2 \mu} \partial_{t}^{3} v^{\prime} e^{S_{0}}\right)^{\prime}\right] \\
= & -\frac{1}{\gamma} \omega^{1 / 2+\mu} \partial_{t}^{5} v-(\gamma+1) \frac{\omega^{3 / 2+\mu} \partial_{t}^{3} v^{\prime} e^{S_{0}} \eta^{\prime \prime}}{\left(\eta^{\prime}\right)^{\gamma+2}} \\
& +\sum_{i=1}^{3} c_{i} \omega^{-(1 / 2+\mu)}\left(\omega^{2+2 \mu} \partial_{t}^{i} \frac{1}{\left(\eta^{\prime}\right)^{\gamma+1}} \partial_{t}^{3-i} v^{\prime} e^{S_{0}}\right)^{\prime} \\
& +\omega^{-(1 / 2+\mu)}\left[\left(1-\frac{1}{\left(\eta^{\prime}\right)^{\gamma+1}}\right)\left(\omega^{2+2 \mu} \partial_{t}^{3} v^{\prime} e^{S_{0}}\right)^{\prime}\right] .
\end{aligned}
$$

Using Lemma 2.1 and fundamental theorem of calculus for the terms on the right-hand side of (4.19], we obtain that for any $t \in\left[0, T^{\varepsilon}\right]$,

$$
\begin{aligned}
\sup _{[0, t]}\left\|\omega^{-(1 / 2+\mu)}\left(\omega^{2+2 \mu} \partial_{t}^{3} v^{\prime} e^{S_{0}}\right)^{\prime}\right\|_{0} \leq & C \sup _{[0, t]}\left\|\omega^{1 / 2+\mu} \partial_{t}^{5} v\right\|_{0}+C \sup _{[0, t]}\left\|\frac{\omega^{3 / 2+\mu} \partial_{t}^{3} v^{\prime} e^{S_{0}} \eta^{\prime \prime}}{\eta^{\prime(\gamma+2)}}\right\|_{0} \\
& +C \sup _{[0, t]} \sum_{i=1}^{3}\left\|\omega^{-(1 / 2+\mu)}\left(\omega^{2+2 \mu} \partial_{t}^{i} \frac{1}{\left(\eta^{\prime}\right)^{\gamma+1}} \partial_{t}^{3-i} v^{\prime} e^{S_{0}}\right)^{\prime}\right\|_{0} \\
& +C \sup _{[0, t]}\left\|\omega^{-(1 / 2+\mu)}\left[\left(1-\frac{1}{\left(\eta^{\prime}\right)^{\gamma+1}}\right)\left(\omega^{2+2 \mu} \partial_{t}^{3} v^{\prime} e^{S_{0}}\right)^{\prime}\right]\right\|_{0} \\
= & : \sum_{j=5}^{8} I_{j} .
\end{aligned}
$$


We estimate each term on the right-hand side of (4.20). Noting that $-\frac{1}{4}<\mu \leq 0$, as $2 \leq \gamma<3$, and using the first term of the estimate (4.14), one has, for each $t \in\left[0, T_{\varepsilon}\right]$,

$$
I_{5}=\sup _{[0, t]}\left\|\omega^{1 / 2+\mu} \partial_{t}^{5} v\right\|_{0} \leq C \hat{E}^{\alpha / 2}\left(\hat{M}_{0}+C t P\left(\sup _{[0, t]} \hat{E}\right)\right) .
$$

The second term $I_{6}$ on the right-hand side of (4.20) can be estimated as

$$
\begin{aligned}
I_{6} & =\left\|\frac{\omega^{3 / 2+\mu} \partial_{t}^{3} v^{\prime} \eta^{\prime \prime} e^{S_{0}}}{\left(\eta^{\prime}\right)^{\gamma+2}}\right\|_{0} \leq C\left\|\omega^{3 / 2+\mu} \partial_{t}^{3} v^{\prime}\right\|_{\infty}\left\|\int_{0}^{t} v^{\prime \prime}\right\|_{0} \\
& \leq C t\left(\left\|\omega^{1 / 2+\mu} \omega^{\prime} \partial_{t}^{3} v^{\prime}\right\|_{0}+\left\|\omega^{3 / 2+\mu} \partial_{t}^{3} v^{\prime \prime}\right\|_{0}\right)\left\|v^{\prime \prime}\right\|_{0} \leq C t P\left(\sup _{[0, t]} \hat{E}\right)
\end{aligned}
$$

For the third term, we have,

$$
\begin{aligned}
I_{7}= & \sum_{i=1}^{3}\left\|\omega^{-(1 / 2+\mu)}\left(\omega^{2+2 \mu} \partial_{t}^{i} \frac{1}{\left(\eta^{\prime}\right)^{\gamma+1}} \partial_{t}^{3-i} v^{\prime} e^{S_{0}}\right)^{\prime}\right\|_{0} \\
\leq & \left.C\left\|\omega^{3 / 2+\mu}\left(v^{\prime}\right)^{3} v^{\prime \prime}\right\|\right|_{0}+C|| \omega^{3 / 2+\mu}\left(\left|\partial_{t} v^{\prime \prime}\left(v^{\prime}\right)^{2}\right|+\left|\partial_{t} v^{\prime} v^{\prime \prime} v^{\prime}\right|\right) \|_{0} \\
& +C\left\|\omega^{3 / 2+\mu}\left(\left|\partial_{t}^{2} v^{\prime \prime} v^{\prime}\right|+\left|\partial_{t}^{2} v^{\prime} v^{\prime \prime}\right|\right)\right\|_{0}+C|| \omega^{3 / 2+\mu} \partial_{t} v^{\prime} \partial_{t} v^{\prime \prime} \|_{0} \\
& +C\left\|\left(\omega^{1 / 2+\mu} \omega^{\prime}+\omega^{3 / 2}+\omega^{3 / 2+\mu} \eta^{\prime \prime}\right)\left|\left(v^{\prime}\right)^{4} \|\right|_{0}\right. \\
& +C\left\|\left(\omega^{1 / 2+\mu} \omega^{\prime}+\omega^{3 / 2+\mu}+\omega^{3 / 2+\mu} \eta^{\prime \prime}\right)\left|\partial_{t} v^{\prime}\left(v^{\prime}\right)^{2} \|\right|_{0}\right. \\
& +C\left\|\left(\omega^{1 / 2+\mu} \omega^{\prime}+\omega^{3 / 2+\mu}+\omega^{3 / 2+\mu} \eta^{\prime \prime}\right)\left|\partial_{t}^{2} v^{\prime} v^{\prime}\right|\right\|_{0} \\
& +C\left\|\left(\omega^{1 / 2+\mu} \omega^{\prime}+\omega^{3 / 2+\mu}+\omega^{3 / 2+\mu} \eta^{\prime \prime}\right) \mid\left(\partial_{t} v^{\prime}\right)^{2}\right\|_{0} \\
= & \sum_{i=1}^{8} I_{7 i}
\end{aligned}
$$

where we have used the fact $1 / 2 \leq \eta^{\prime} \leq 3 / 2$ and (2.20). To obtain the desired estimates, we shall use the following form of estimates:

$$
\begin{aligned}
\sum_{j=1}^{2}\left\{\left\|\omega^{3 / 2+\mu} \partial_{t}^{4-2 j} \partial_{x}^{j+1} v\right\|_{0}^{2}\right. & \left.+\sum_{i=1}^{j}\left\|\omega^{1 / 2+\mu} \partial_{t}^{4-2 j} \partial_{x}^{i} v\right\|_{0}^{2}+\sum_{i=-1}^{j}\left\|\omega^{1+\mu} \partial_{t}^{3-2 j} \partial_{x}^{i+1} v\right\|_{0}^{2}\right\} \\
\leq & C \sum_{j=1}^{2}\left\{\left\|\omega^{3 / 2+\mu} u_{4-2 j}^{(j+1)}+\int_{0}^{t} \omega^{3 / 2+\mu} \partial_{t}^{5-2 j} \partial_{x}^{j+1} v\right\|_{0}^{2}\right. \\
& +\sum_{i=1}^{j}\left\|\omega^{1 / 2+\mu} u_{4-2 j}^{(i)}+\int_{0}^{t} \omega^{1 / 2+\mu} \partial_{t}^{5-2 j} \partial_{x}^{i} v\right\|_{0}^{2} \\
& \left.+\sum_{i=-1}^{j}\left\|\omega^{1+\mu} u_{3-2 j}^{(i+1)}+\int_{0}^{t} \omega^{1 / 2+\mu} \partial_{t}^{4-2 j} \partial_{x}^{i+1} v\right\|_{0}^{2}\right\} \\
\leq & \hat{M}_{0}+C t P\left(\sup _{[0, t]} \hat{E}\right) .
\end{aligned}
$$


For $I_{71}$, using (2.12), we have

$$
\begin{aligned}
I_{71} & =\left\|\omega^{3 / 2+\mu}\left(v^{\prime}\right)^{3} v^{\prime \prime}\right\|_{0} \leq\left\|\omega^{3 / 2+\mu} v^{\prime \prime}\right\|_{\infty}\left\|v^{\prime}\right\|_{L^{6}}^{3} \\
& \leq C\left\|\omega^{3 / 2+\mu} v^{\prime \prime}\right\|_{1}\left\|v^{\prime}\right\|_{1 / 2}^{3} \leq \hat{M}_{0}+C t P\left(\sup _{[0, t]} \hat{E}\right),
\end{aligned}
$$

where we have used the physical vacuum condition (1.7) and (4.24), as well as the following two estimates:

$$
\left\|\omega^{3 / 2+\mu} v^{\prime \prime}\right\|_{\infty} \leq\left\|\omega^{3 / 2+\mu} v^{\prime \prime}\right\|_{1} \leq C\left\|\omega^{1 / 2+\mu} \omega^{\prime} v^{\prime \prime}+\omega^{3 / 2+\mu} v^{\prime \prime \prime}\right\|_{0} \leq \hat{M}_{0}+C t P\left(\sup _{[0, t]} \hat{E}\right)
$$

and

$$
\left\|v^{\prime}\right\|_{1 / 2} \leq\left\|u_{0}^{\prime}+\int_{0}^{t} \partial_{t} v^{\prime}\right\|_{1 / 2} \leq C \hat{M}_{0}+C t P\left(\sup _{[0, t]} \hat{E}\right) .
$$

For $I_{72}$, using (4.26), we have

$$
\begin{aligned}
I_{72} & =\left\|\omega^{3 / 2+\mu}\left(\partial_{t} v^{\prime \prime}\left(v^{\prime}\right)^{2}+\partial_{t} v^{\prime} v^{\prime \prime} v^{\prime}\right)\right\|_{0} \\
& \leq\left. C\left\|\omega^{\frac{3}{2}+\mu} \partial_{t} v^{\prime \prime}\right\|\right|_{L^{4}}\left\|v^{\prime}\right\|_{L^{8}}^{2}+C\left\|\omega^{\frac{3}{2}+\mu} v^{\prime \prime}\right\|_{\infty}\left\|\partial_{t} v^{\prime}\right\|\left\|_{0}\right\| v^{\prime} \|_{\infty} \\
& \leq\left. C\left\|\omega^{\frac{3}{2}+\mu} \partial_{t} v^{\prime \prime}||_{0}^{1 / 2}\right\| \omega^{\frac{3}{2}+\mu} \partial_{t} v^{\prime \prime}\left\|\left.\right|_{1} ^{1 / 2}\right\| v^{\prime}\left\|_{1 / 2}^{2}+C\right\| \omega^{\frac{3}{2}+\mu} v^{\prime \prime}\left\|_{1}\right\| \partial_{t} v^{\prime}\|\|_{0}\left\|v^{\prime}\right\|\right|_{1 / 2} ^{1 / 2}\left\|v^{\prime}\right\|_{1}^{1 / 2} \\
& \leq\left(1+\hat{E}^{1 / 4}\right)\left(\hat{M}_{0}+t P\left(\sup _{[0, t]} \hat{E}\right)\right)
\end{aligned}
$$

where we used (4.24) to derive the following two estimates

$$
\begin{gathered}
\left\|\omega^{\frac{3}{2}+\mu} \partial_{t} v^{\prime \prime}\right\|_{0}^{1 / 2} \leq C\|\omega\|_{\infty}^{\frac{1}{4}}\left\|\omega^{1+\mu} \partial_{t} v^{\prime \prime}\right\|_{0}^{1 / 2} \leq \hat{M}_{0}+t P\left(\sup _{[0, t]} \hat{E}\right), \\
\left\|\partial_{t} v^{\prime}\right\|_{0} \leq C\left\|u_{1}^{\prime}+\int_{0}^{t} \partial_{t}^{2} v^{\prime}\right\|_{0} \leq \hat{M}_{0}+C t P\left(\sup _{[0, t]} \hat{E}\right),
\end{gathered}
$$

and used the physical vacuum condition (1.7) to get

$$
\left\|\omega^{3 / 2+\mu} \partial_{t} v^{\prime \prime}\right\|_{1}^{1 / 2}=\left\|\omega^{1 / 2+\mu} \omega^{\prime} \partial_{t} v^{\prime \prime}+\omega^{3 / 2+\mu} \partial_{t} v^{\prime \prime \prime}\right\|_{0}^{1 / 2} \leq C \hat{E}^{1 / 4}
$$

Using (4.24) to estimate $\left\|\omega^{3 / 2+\mu} \partial_{t}^{2} v^{\prime \prime} \mid\right\|_{0},\left\|\omega^{1 / 2+\mu} \partial_{t}^{2} v^{\prime}\right\|_{0}$ and $\left\|\omega^{1 / 2+\mu} v^{\prime \prime}\right\|_{0}$, one has,

$$
\begin{aligned}
I_{73} & =\left\|\omega^{3 / 2+\mu}\left(\partial_{t}^{2} v^{\prime \prime} v^{\prime}+\partial_{t}^{2} v^{\prime} v^{\prime \prime}\right)\right\|_{0} \\
& \leq C\left\|\omega^{3 / 2+\mu} \partial_{t}^{2} v^{\prime \prime}\right\|\left\|_{0}\right\| v^{\prime}\left\|_{\infty}+C\right\| \omega^{1 / 2+\mu} \partial_{t}^{2} v^{\prime}\|\|_{0}\left\|\omega v^{\prime \prime}\right\|_{\infty} \\
& \leq\left(\hat{E}^{3 / 8}+1\right)\left(\hat{M}_{0}+C t P\left(\sup _{[0, t]} \hat{E}\right)\right)
\end{aligned}
$$


where we have used the fact that

$$
\begin{aligned}
\left\|\omega v^{\prime \prime}\right\|_{\infty} & \leq C\|\omega\|^{-\mu / 4}\left\|\omega^{1+\mu} v^{\prime \prime} \mid\right\|_{0}^{1 / 4}\left\|v^{\prime \prime}+\omega v^{\prime \prime \prime}\right\|_{0}^{3 / 4} \\
& \leq C \| \omega^{1 / 2+\mu} v^{\prime \prime}||_{0}^{1 / 4}\left(\left\|v^{\prime \prime}\right\|_{0}^{3 / 4}+\|\omega\|^{-3 \mu / 4}\left\|\omega^{1+\mu} v^{\prime \prime \prime}\right\|_{0}^{3 / 4}\right) \\
& \leq \hat{E}^{3 / 8}\left(\hat{M}_{0}+C t P\left(\sup _{[0, t]} \hat{E}\right)\right) .
\end{aligned}
$$

For $I_{74}$, using (4.27) and (4.24) to estimate $\left\|\omega^{1+\mu} \partial_{t} v^{\prime \prime}\right\| \|_{0}$, we have

$$
\begin{aligned}
& I_{74}=\left\|\omega^{3 / 2+\mu} \partial_{t} v^{\prime} \partial_{t} v^{\prime \prime}\right\|_{0} \leq C\left\|\omega^{3 / 2+\mu} \partial_{t} v^{\prime \prime}\right\|_{\infty}\left\|\partial_{t} v^{\prime}\right\|_{0} \\
& \leq\left. C\left\|\omega^{3 / 2+\mu} \partial_{t} v^{\prime \prime}\right\|\right|_{0} ^{1 / 4}\left\|\omega^{3 / 2+\mu} \partial_{t} v^{\prime \prime}\right\|_{1}^{3 / 4}\left\|\partial_{t} v^{\prime}\right\|_{0} \\
& \leq C\|\omega\|\left\|_{\infty}^{1 / 8}\right\| \omega^{1+\mu} \partial_{t} v^{\prime \prime}\left\|_{0}^{1 / 4}\right\| \omega^{1 / 2+\mu} \partial_{t} v^{\prime \prime}+\omega^{3 / 2} \partial_{t} v^{\prime \prime \prime}\left\|\left.\right|_{0} ^{3 / 4}\right\| \partial_{t} v^{\prime} \|_{0} \\
& \leq \hat{E}^{\frac{3}{8}}\left(\hat{M}_{0}+C t P\left(\sup _{[0, t]} \hat{E}\right)\right) .
\end{aligned}
$$

For $I_{75}$, using (4.26) to estimate $\left\|v^{\prime}\right\|_{L^{8}}$, we have

$$
\begin{aligned}
I_{75} & =\left\|\left(\omega^{1 / 2+\mu} \omega^{\prime}+\omega^{3 / 2+\mu}+\omega^{3 / 2+\mu} \eta^{\prime \prime}\right)\left(v^{\prime}\right)^{4}\right\|_{0} \\
& \leq C\left\|\omega^{1 / 2+\mu}\right\|_{\infty}\left\|v^{\prime}\right\|_{L^{8}}^{4}+C\left\|\int_{0}^{t} \omega^{1+\mu} v^{\prime \prime}\right\|\left\|_{0}\right\| v^{\prime}\left\|_{\infty}^{4}\right\| \omega \|_{\infty}^{3 / 2} \\
& \leq \hat{M}_{0}+C t P\left(\sup _{[0, t]} \hat{E}\right) .
\end{aligned}
$$

Similarly, using (4.26) and (4.27) again, one has

$$
\begin{aligned}
I_{76} & =\left\|\left(\omega^{1 / 2+\mu} \omega^{\prime}+\omega^{3 / 2+\mu}+\omega^{3 / 2+\mu} \eta^{\prime \prime}\right) \partial_{t} v^{\prime}\left(v^{\prime}\right)^{2}\right\|_{0} \\
& \leq\left. C\left\|\omega^{1 / 2+\mu} \partial_{t} v^{\prime}\right\|\right|_{L^{4}}\left\|v^{\prime}\right\|_{L^{8}}^{2}+\left.C\left\|\int_{0}^{t} \omega^{3 / 2+\mu} v^{\prime \prime}\right\|_{L^{4}}\left\|\partial_{t} v^{\prime}\right\|\right|_{L^{4}}\left\|v^{\prime}\right\|_{\infty}^{2} \\
& \leq \hat{M}_{0}+C t P\left(\sup _{[0, t]} \hat{E}\right),
\end{aligned}
$$

where we have used (2.13), the method similar to (4.12) to deal with $\left\|\omega^{1 / 2+\mu} \partial_{t} v^{\prime}\right\|_{1 / 2}$, and (4.24) to estimate $\left\|\omega^{1+\mu} \partial_{t} v^{\prime}\right\|_{0}$, as well as

$$
\left\|\omega^{1 / 2+\mu} \partial_{t} v^{\prime}\right\|_{L^{4}} \leq C\left\|\omega^{1 / 2+\mu} \partial_{t} v^{\prime}\right\|_{1 / 2} \leq C\left\|\omega^{\mu} \partial_{t} v^{\prime}+\omega^{1+\mu} \partial_{t} v^{\prime \prime}\right\|_{0} \leq \hat{M}_{0}+C t P\left(\sup _{[0, t]} \hat{E}\right)
$$

and

$$
\left\|\int_{0}^{t} \omega^{3 / 2+\mu} v^{\prime \prime}\right\|_{L^{4}} \leq\left\|\int_{0}^{t} \omega^{3 / 2+\mu} v^{\prime \prime}\right\|_{1} \leq\left\|\int_{0}^{t}\left(\omega^{1 / 2+\mu} \omega^{\prime} v^{\prime \prime}+\omega^{3 / 2+\mu} v^{\prime \prime \prime}\right)\right\|_{0} \leq C t P\left(\sup _{[0, t]} \hat{E}\right) .
$$


For $I_{77}$, using (4.24) to deal with $\left\|\omega^{1 / 2+\mu} \partial_{t}^{2} v^{\prime}\right\|_{0}$ and (4.26), we have

$$
\begin{aligned}
I_{77} & =\left\|\left(\omega^{1 / 2+\mu} \omega^{\prime}+\omega^{3 / 2+\mu}+\omega^{3 / 2+\mu} \eta^{\prime \prime}\right) \partial_{t}^{2} v^{\prime} v^{\prime}\right\|_{0} \\
& \leq C\left\|\omega^{1 / 2+\mu} \partial_{t}^{2} v^{\prime}\right\|\left\|_{0}\right\| v^{\prime} \|_{\infty}\left(1+\left\|\int_{0}^{t} v^{\prime \prime}\right\|_{0}\right) \\
& \leq \hat{E}^{1 / 4}\left(\hat{M}_{0}+C t P\left(\sup _{[0, t]} \hat{E}\right)\right)+C t P\left(\sup _{[0, t]} \hat{E}\right) .
\end{aligned}
$$

For $I_{78}$, using 4.27, we have

$$
\begin{aligned}
I_{78}= & \left\|\left(\omega^{1 / 2+\mu} \omega^{\prime}+\omega^{3 / 2+\mu}+\omega^{3 / 2+\mu} \eta^{\prime \prime}\right)\left(\partial_{t} v^{\prime}\right)^{2}\right\|_{0} \\
\leq & C\left\|\omega^{1 / 2+\mu} \partial_{t} v^{\prime}\right\|_{\infty}\left\|\partial_{t} v^{\prime}\right\|_{0}+C\left\|\omega^{3 / 2+\mu} \partial_{t} v^{\prime}\right\|_{\infty}\left\|\partial_{t} v^{\prime}\right\|_{0} \\
& +C\left\|\int_{0}^{t} \omega^{3 / 2+\mu} v^{\prime \prime}\right\|_{\infty}\left\|\partial_{t} v^{\prime}\right\|_{L^{4}}^{2} \\
\leq & C\left\|\omega^{1 / 2+\mu} \partial_{t} v^{\prime}\right\|_{3 / 4}\left\|\partial_{t} v^{\prime}\right\|_{0}+C\left\|\omega^{3 / 2+\mu} \partial_{t} v^{\prime}\right\|_{1}\left\|\partial_{t} v^{\prime}\right\|_{0} \\
& +C t\left\|\omega^{3 / 2+\mu} v^{\prime \prime}\right\|_{1}\left\|\partial_{t} v^{\prime}\right\|_{1 / 2}^{2} \\
\leq & C\left\|u_{1}^{\prime}+\int_{0}^{t} \partial_{t}^{2} v^{\prime}\right\|_{0}^{1-\alpha}\left\|\left(\omega^{1 / 2} \partial_{t} v^{\prime}\right)^{\prime}\right\|_{L^{r}}^{\alpha}\left\|\partial_{t} v^{\prime}\right\|_{0} \\
& +C\left(\left\|\omega^{1 / 2+\mu} \omega^{\prime} \partial_{t} v^{\prime}\right\|_{0}+\left\|\omega^{1 / 2+\mu} \omega \partial_{t} v^{\prime \prime}\right\| \|_{0}\right)\left\|\partial_{t} v^{\prime}\right\|_{0} \\
& +C t\left(\left\|\omega^{1 / 2+\mu} \omega^{\prime} v^{\prime \prime}\right\|\left\|_{0}+\right\| \omega^{3 / 2+\mu} v^{\prime \prime \prime}\|\|_{0}\right)\left\|\partial_{t} v^{\prime}\right\|_{1 / 2}^{2} \\
\leq & \left(\hat{E}^{\frac{\alpha}{2}}+1\right)\left(\hat{M}_{0}+C t P\left(\sup _{[0, t]} \hat{E}\right)\right)+C t P\left(\sup _{[0, t]} \hat{E}\right)
\end{aligned}
$$

where $\alpha_{0}<\alpha<1$ with $\frac{3}{4} \leq \alpha_{0}=\frac{3}{4(1+\mu)}=\frac{3}{2}\left(1-\frac{1}{\gamma}\right)<1$ as $2 \leq \gamma<3$ and $\frac{1}{r}=\frac{3}{2}-\frac{3}{4 \alpha}$, and we have used the following estimate:

$$
\begin{aligned}
\left\|\left(\omega^{1 / 2+\mu} \partial_{t} v^{\prime}\right)^{\prime}\right\| & \leq C\left\|\frac{\partial_{t} v^{\prime}}{\omega^{1 / 2+\mu}}\right\|\left\|_{L^{r}}+C\right\| \omega^{1 / 2+\mu} \partial_{t} v^{\prime \prime} \|_{0} \\
& \leq\left. C\left\|\frac{1}{\omega^{1 / 2-\mu}}\right\|\right|_{L^{\beta}}\left\|\partial_{t} v^{\prime}\right\| L_{L^{\beta^{\prime}}}+C\left\|\omega^{1 / 2+\mu} \partial_{t} v^{\prime \prime}\right\|_{0} \\
& \leq C\left\|\frac{1}{\omega^{1 / 2-\mu}}\right\|\left\|_{L^{\beta}}\right\| \partial_{t} v^{\prime}\left\|_{1 / 2}+C\right\| \omega\left\|_{\infty}^{-\mu}\right\| \omega^{1 / 2+\mu} \partial_{t} v^{\prime \prime} \|_{0},
\end{aligned}
$$

where, to ensure $\left\|\frac{1}{\omega^{1 / 2-\mu}}\right\|_{L^{\beta}}$ be meaningful, we need $\left.\beta\left(\frac{1}{2}-\mu\right)\right)<1$, so we choose $\frac{1}{\beta} \in\left(\frac{1}{2}-\mu, \frac{1}{r}\right)$, and $\frac{1}{r}=\frac{1}{\beta}+\frac{1}{\beta^{\prime}}$, with $\beta^{\prime}>1$ and $\left\|\partial_{t} v^{\prime}\right\|_{L^{\beta^{\prime}}} \leq\left\|\partial_{t} v^{\prime}\right\|_{1 / 2}$ in (2.8). This analysis is different from the isentropic case of $\gamma=2$. Substituting (4.25)-(4.28) into (4.23), we have

$$
I_{7} \leq\left(\hat{E}^{\frac{\alpha}{2}}+1\right)\left(\hat{M}_{0}+C t P\left(\sup _{[0, t]} \hat{E}\right)\right)+C t P\left(\sup _{[0, t]} \hat{E}\right) .
$$


Now for the last term on the right-hand side of (4.20), due to

$$
1-\frac{1}{\eta^{\prime(\gamma+1)}}=(\gamma+1) \int_{0}^{t} \frac{v^{\prime}}{\eta^{\prime(\gamma+2)}},
$$

it can be estimated as

$$
\begin{aligned}
I_{8}= & \left\|\omega^{-(1 / 2+\mu)}\left[\left(1-\frac{1}{\left(\eta^{\prime}\right)^{\gamma+1}}\right)\left(\omega^{2+2 \mu} \partial_{t}^{3} v^{\prime} e^{S_{0}}\right)^{\prime}\right]\right\|_{0} \\
\leq & C\left\|(\gamma+1) \int_{0}^{t} \frac{v^{\prime}}{\left(\eta^{\prime}\right)^{\gamma+2}}\right\|_{\infty}\left(\left\|\omega^{1 / 2+\mu} \partial_{t}^{3} v^{\prime}\right\|\left\|_{0}\right\| \omega^{\prime}\left\|_{\infty}+\right\| \omega^{1 / 2+\mu} \partial_{t}^{3} v^{\prime \prime}\left\|_{0}\right\| \omega \|_{\infty}^{1 / 2}\right. \\
& \left.+\left\|\omega^{1 / 2+\mu} \partial_{t}^{3} v^{\prime}\right\|_{0}\|\omega\|_{\infty}^{1 / 2}\right) \leq C t P\left(\sup _{[0, t]} \hat{E}\right) .
\end{aligned}
$$

Substituting (4.21), 4.22), 4.30) and (4.32) into (4.20) leads to

$$
\begin{aligned}
& \sup _{t \in[0, t]}\left\|\omega^{-(1 / 2+\mu)}\left(\omega^{2+2 \mu} \partial_{t}^{3} v^{\prime} e^{S_{0}}\right)^{\prime}\right\|_{0}^{2} \\
& \quad \leq C\left(\hat{E}^{\alpha / 2}+1\right)^{2}\left(\hat{M}_{0}+C t P\left(\sup _{[0, t]} \hat{E}\right)\right)+C t P\left(\sup _{[0, t]} \hat{E}\right) .
\end{aligned}
$$

Expanding the left-hand side of (4.33) and using physical vacuum condition (1.7), we have

$$
\begin{aligned}
& \sup _{t \in[0, t]}\left\|\omega^{-(1 / 2+\mu)}\left(\omega^{2+2 \mu} \partial_{t}^{3} v^{\prime} e^{S_{0}}\right)^{\prime}\right\|_{0}^{2} \geq 4(1+\mu)^{2}\left\|\omega^{1 / 2+\mu} \omega^{\prime} \partial_{t}^{3} v^{\prime} e^{S_{0}}\right\|_{0}^{2}+\left\|\omega^{3 / 2+\mu} \partial_{t}^{3} v^{\prime \prime} e^{S_{0}}\right\|_{0}^{2} \\
& +\left\|\omega^{3 / 2+\mu} \partial_{t}^{3} v^{\prime} e^{S_{0}} S_{0}^{\prime} \omega^{-\mu}\right\|_{0}^{2} \\
& +4(1+\mu) \int_{I} \omega^{2+\mu} \partial_{t}^{3} v^{\prime} \partial_{t}^{3} v^{\prime \prime} \omega^{\prime} \exp 2 S_{0} \\
& +4(1+\mu) \int_{I} \omega^{2+2 \mu}\left|\partial_{t}^{3} v^{\prime}\right|^{2} \omega^{\prime} \exp 2 S_{0} S_{0}^{\prime} \\
& +2 \int_{I} \omega^{3+2 \mu} \partial_{t}^{3} v^{\prime} \partial_{t}^{3} v^{\prime \prime} \exp 2 S_{0} \\
& \geq C\left\|\omega^{1 / 2+\mu} \partial_{t}^{3} v^{\prime}||_{0}^{2}+C\right\| \omega^{3 / 2+\mu} \partial_{t}^{3} v^{\prime \prime} \|_{0}^{2} \\
& -C\left(1+\|\omega\|_{\infty}\right)\left(\left\|\omega^{1+\mu} \partial_{t}^{3} v^{\prime}\right\|_{0}^{2}+\left\|\omega^{1+\mu} \partial_{t}^{3} v^{\prime}\right\|_{0}^{2}\right) \\
& \geq C\left\|\omega^{1 / 2+\mu} \partial_{t}^{3} v^{\prime}\right\|_{0}^{2}+C\left\|\omega^{3 / 2+\mu} \partial_{t}^{3} v^{\prime \prime}\right\|_{0}^{2} \\
& -\hat{M}_{0}-C t P\left(\sup _{[0, t]} \hat{E}\right) \text {, }
\end{aligned}
$$

and thus, by 4.33),

$$
\begin{aligned}
& \sup _{[0, t]}\left(\left\|\omega^{1 / 2+\mu} \partial_{t}^{3} v^{\prime}\right\|_{0}^{2}+\left\|\omega^{3 / 2+\mu} \partial_{t}^{3} v^{\prime \prime}\right\|_{0}^{2}\right) \\
& \quad \leq C\left(\hat{E}^{\alpha}+1\right)\left(\hat{M}_{0}+C t P\left(\sup _{[0, t]} \hat{E}\right)\right)+C t P\left(\sup _{[0, t]} \hat{E}\right) .
\end{aligned}
$$


Choosing the multiplier $\omega^{-(1 / 2+\mu)}$ and letting the first term $\omega^{1+2 \mu} \partial_{t}^{k} v$ in $g$ be $\omega^{1+2 \mu} \partial_{t} v^{\prime}$, using the same method as that of proving (4.17), we can prove (4.18).

Similarly, multiplying both sides of (4.16) by $\omega^{-\mu}$ and replacing the first term $\omega^{1+2 \mu} \partial_{t}^{k} v$ in $g$ by $\omega^{1+2 \mu} \partial_{t}^{2} v, \omega^{1+2 \mu} \partial_{t}^{2} v^{\prime}$ and $\omega^{1+2 \mu} v^{\prime}$, respectively, one has

Proposition 4.4. For $2 \leq \gamma<3$, there exists some $\alpha \in(0,1)$, such that the following estimate holds,

$$
\begin{aligned}
& \sup _{[0, t]}\left(\left.\left\|\omega^{1+\mu} \partial_{t}^{2} v^{\prime}\right\|\right|_{0} ^{2}+\left.\left\|\omega^{2+\mu} \partial_{t}^{2} v^{\prime \prime}\right\|\right|_{0} ^{2}+\left\|\omega^{2+\mu} \partial_{t}^{2} v^{\prime \prime \prime}||_{0}^{2}+\right\| \omega^{1+\mu} \partial_{t}^{2} v^{\prime \prime}\left\|_{0}^{2}+\right\| \omega^{1+\mu} v^{\prime \prime} \|_{0}^{2}\right. \\
& \left.+\left.\left\|\omega^{1+\mu} v^{\prime \prime \prime}\right\|\right|_{0} ^{2}+\left\|\omega^{2+\mu} \partial_{x}^{4} v\right\|_{0}^{2}\right) \\
& \leq C\left(\hat{E}^{\alpha}+1\right)\left(\hat{M}_{0}+t P\left(\sup _{[0, t]} \hat{E}\right)\right)+C t P\left(\sup _{[0, t]} \hat{E}\right) .
\end{aligned}
$$

\section{$5 \quad$ Uniform estimates of $v^{\varepsilon}$ for $1<\gamma<2$}

In this section, we shall establish the uniform estimates for the case $1<\gamma<2$. As noted in [32], the constant $\gamma$ affects the degeneracy rate near the vacuum boundary, since $\rho_{0}$ looks to be the coefficient of $\partial_{t} v$ in $(2.5)_{1}$ and the physical vacuum condition indicates that

$$
\rho_{0}(\eta) \sim \operatorname{dist}(\eta, \partial I)^{\frac{1}{\gamma-1}}, \quad \eta \rightarrow \partial I
$$

Smaller values of $\gamma$ cause more degeneracy of $(2.5)_{1}$ near the vacuum boundary, then higher-order derivatives in the energy function are needed to control the $H^{2}$-norm and hence the $C^{1}$-norm of $v$. From the embedding inequality (2.13), the higher energy function $\tilde{E}(t)$ defined in (2.15) for $1<\gamma<2$ implies that

$$
\|v\|_{2}^{2} \leq\|v\|_{\frac{l+1}{2}-\left(\frac{1}{2}+\mu\right)}^{2} \leq C \sum_{i=0}^{\frac{l+1}{2}}\left\|\omega^{1 / 2+\mu} \partial_{x}^{i} v\right\|_{0}^{2} \leq C \tilde{E}, \quad l=3+2\left\lceil\frac{1}{2}+\mu\right\rceil .
$$

This suggests that the higher-order energy function $\tilde{E}$ is appropriate for the physical vacuum problem (2.5) when $\gamma \in(1,2)$, and as $\gamma \rightarrow 1$ (which means $\mu \rightarrow \infty$ ) the estimate of $\|v\|_{2}^{2}$ needs infinite higher-order derivatives.

\subsection{Energy estimates}

In order to obtain a series of estimates independent of $\varepsilon$, we first need some energy estimates as in Propositions 4.1,4.2. 
Proposition 5.1. For $\gamma \in(1,2)$, we have the following $\varepsilon$-independent energy estimate for $\partial_{t}^{l+1}$-problem of (3.2),

$$
\begin{aligned}
\left\|\omega^{1 / 2+\mu} \partial_{t}^{l+1} v\right\|_{0}^{2} & +\left\|\omega^{1+\mu} \partial_{t}^{l} v^{\prime}\right\|_{0}^{2}+\left\|\omega^{1+\mu} \partial_{t}^{l} v\right\|_{0}^{2}+\varepsilon \int_{0}^{t}\left\|\omega^{1+\mu} \partial_{t}^{l+1} v^{\prime}\right\|_{0}^{2} \\
& \leq \tilde{E}^{\alpha}\left(\tilde{M}_{0}+C t P\left(\sup _{[0, t]} \tilde{E}\right)\right)
\end{aligned}
$$

with $\tilde{M}_{0}=P(\tilde{E}(0))$ and $P(\cdot)$ some polynomial function.

Proof. Similar to the derivation of (4.6), we first take $(l+1)$-th time derivative of equation (3.2 $)_{1}$, then multiply it by $\partial_{t}^{l+1} v$ and integrate this resulting equation with respect to time and space to get

$$
\frac{1}{2} \frac{d}{d t} \int_{I} \omega^{1+2 \mu}\left|\partial_{t}^{l+1} v\right|^{2}-\int_{I} \partial_{t}^{l+1}\left(\frac{\omega^{2+2 \mu}}{\left(\eta^{\prime}\right)^{\gamma}}\right) \partial_{t}^{l+1} v^{\prime} e^{S_{0}}+\varepsilon \int_{I}\left|\omega^{1+\mu} \partial_{t}^{l+1} v^{\prime}\right|^{2} e^{S_{0}}=0
$$

Using similar argument as in (4.7) to deal with the second item on the left-hand side of (5.1), we get

$$
\begin{aligned}
\frac{1}{2} \int_{I} \omega^{1+2 \mu}\left|\partial_{t}^{l+1} v\right|^{2} & +\frac{\gamma}{2} \int_{I} \frac{\omega^{2+2 \mu}}{\left(\eta^{\prime}\right)^{\gamma+1}}\left|\partial_{t}^{l} v^{\prime}\right|^{2} e^{S_{0}}+\varepsilon \int_{0}^{t} \int_{I}\left|\omega^{1+\mu} \partial_{t}^{l+1} v^{\prime}\right|^{2} \\
= & \frac{1}{2} \int_{I} \omega^{1+2 \mu}\left|\partial_{t}^{l+1} v_{0}\right|^{2}+\frac{\gamma}{2} \int_{I} \frac{\omega^{2+2 \mu}}{\left(\eta^{\prime}\right)^{\gamma+1}}\left|\partial_{t}^{l} v_{0}^{\prime}\right|^{2} e^{S_{0}} \\
& -\frac{\gamma}{\gamma+1} \int_{0}^{t} \int_{I} \omega^{2+2 \mu} \frac{v^{\prime}}{\left(\eta^{\prime}\right)^{\gamma+2}}\left|\partial_{t}^{l} v^{\prime}\right|^{2} e^{S_{0}} \\
& -\sum_{i=1}^{l} c_{i} \int_{0}^{t} \int_{I} \omega^{2+2 \mu} \partial_{t}^{i} \frac{1}{\left(\eta^{\prime}\right)^{\gamma+1}} \partial_{t}^{l-i} v^{\prime} \partial_{t}^{l+1} v^{\prime} e^{S_{0}} \\
= & : \sum_{i=1}^{4} I_{i}
\end{aligned}
$$

It is obvious that $I_{1}, I_{2}$ can be controlled by $\tilde{M}_{0}$. Now we estimate $I_{3}$ and $I_{4}$. Similar to (4.9), we have

$$
\begin{aligned}
I_{3} & =-\frac{\gamma}{\gamma+1} \int_{0}^{t} \int_{I} \omega^{2+2 \mu} \frac{v^{\prime}}{\left(\eta^{\prime}\right)^{\gamma+2}}\left|\partial_{t}^{l} v^{\prime}\right|^{2} e^{S_{0}} \\
& \leq C \int_{0}^{t}\left\|v^{\prime}\right\|_{\infty}\left\|\omega^{1+\mu} \partial_{t}^{l} v^{\prime}\right\|_{0}^{2} \leq C \int_{0}^{t}\|v\|_{2}\left\|\omega^{1+\mu} \partial_{t}^{l} v^{\prime}\right\|_{0}^{2} \\
& \leq C t P\left(\sup _{[0, t]} \tilde{E}\right) .
\end{aligned}
$$


Using integrating by parts in time, we have

$$
\begin{aligned}
I_{4}= & -\sum_{\alpha=1}^{l} c_{i} \int_{0}^{t} \int_{I} \omega^{2+2 \mu} \partial_{t}^{i} \frac{1}{\left(\eta^{\prime}\right)^{\gamma+1}} \partial_{t}^{l-i} v^{\prime} \partial_{t}^{l+1} v^{\prime} e^{S_{0}} \\
= & \sum_{i=1}^{l} c_{i} \int_{0}^{t} \int_{I} \omega^{2+2 \mu}\left(\partial_{t}^{i} \frac{1}{\left(\eta^{\prime}\right)^{\gamma+1}} \partial_{t}^{l-i} v^{\prime}\right)_{t} \partial_{t}^{l} v^{\prime} e^{S_{0}} \\
& -\left.\sum_{i=1}^{l} c_{i} \int_{I} \omega^{2+2 \mu} \partial_{t}^{i} \frac{1}{\left(\eta^{\prime}\right)^{\gamma+1}} \partial_{t}^{l-i} v^{\prime} \partial_{t}^{l} v^{\prime} e^{S_{0}}\right|_{0} ^{t} \\
= & : I_{41}+I_{42} .
\end{aligned}
$$

After detailed computations, using similar analysis as for (4.11)-(4.1) and the definition $\tilde{E}$ in (2.15), $I_{41}$ can be estimated as

$$
\begin{aligned}
I_{41} & \leq C \sum_{i=1}^{l} \int_{0}^{t} \int_{I} \omega^{1+\mu}\left|\left(\partial_{t}^{i} \frac{1}{\left(\eta^{\prime}\right)^{\gamma+1}} \partial_{t}^{k-i} v^{\prime}\right)_{t}\right|\left|\omega^{1+\mu} \partial_{t}^{l} v^{\prime}\right| \\
& \leq C \int_{0}^{t}\left(\left\|\omega^{2+2 \mu} \partial_{t}^{l} v^{\prime} v^{\prime}\right\|_{0}+\left\|\omega^{2+2 \mu} \partial_{t}^{l-1} v^{\prime} \partial_{t} v^{\prime}\right\|_{0}+\cdots\right)\left\|\omega^{2+2 \mu} \partial_{t}^{l} v^{\prime}\right\|_{0} \\
& \leq \tilde{M}_{0}+C t P\left(\sup _{[0, t]} \tilde{E}\right) .
\end{aligned}
$$

To show clearly the idea of proving the estimate (5.5), we will take $1<\gamma=\frac{3}{2}<2$ as an example in the Subsection 5.2. Using the similar method to (4.13), for $0<\alpha<1$, we have

$$
I_{42} \leq \tilde{E}^{\alpha}\left(\tilde{M}_{0}+C t P\left(\sup _{[0, t]} \tilde{E}\right)\right),
$$

where $\tilde{M}_{0}=P(\tilde{E}(0))$. Substituting (5.3)-(5.6) into (5.2), and using the same derivation as (4.15), we can prove Proposition 5.1 .

\subsection{The case of $\gamma=\frac{3}{2}$}

When $\gamma=\frac{3}{2}$, then $\mu=\frac{1}{2}, l=5$. The higher-order energy function $\tilde{E}(t)$ in (2.15) is

$$
\begin{aligned}
\tilde{E}(t)= & \left\|\omega^{1+\mu} \partial_{t}^{5} v^{\prime}(\cdot, t)\right\|_{0}^{2}+\left\|\omega^{1+\mu} \partial_{t}^{5} v(\cdot, t)\right\|_{0}^{2} \\
& +\sum_{j=1}^{3}\left\{\left\|\omega^{3 / 2+\mu} \partial_{t}^{6-2 j} \partial_{x}^{j+1} v(\cdot, t)\right\|_{0}^{2}+\sum_{i=1}^{j}\left\|\omega^{1 / 2+\mu} \partial_{t}^{6-2 j} \partial_{x}^{i} v(\cdot, t)\right\|_{0}^{2}\right\} \\
& +\sum_{j=1}^{2}\left\{\left\|\omega^{2+\mu} \partial_{t}^{5-2 j} \partial_{x}^{j+2} v(\cdot, t)\right\|_{0}^{2}+\sum_{i=-1}^{j}\left\|\omega^{1+\mu} \partial_{t}^{5-2 j} \partial_{x}^{i+1} v(\cdot, t)\right\|_{0}^{2}\right\} .
\end{aligned}
$$

We note that (5.7) also implies the inequality (4.4) which will be usually used in the following elliptic estimates. Now we prove the energy estimate: 
Proposition 5.2. For $\gamma=3 / 2$, there exists a constant $\alpha \in(0,1)$, such that one has the following $\varepsilon$-independent energy estimate on the $\partial_{t}^{6}$ - problem of (3.2),

$$
\begin{aligned}
\left\|\omega^{1 / 2+\mu} \partial_{t}^{6} v\right\|_{0}^{2} & +\left\|\omega^{1+\mu} \partial_{t}^{5} v^{\prime}\right\|_{0}^{2}+\left\|\omega^{1+\mu} \partial_{t}^{5} v\right\|_{0}^{2}+\varepsilon \int_{0}^{t}\left\|\omega^{1+\mu} \partial_{t}^{6} v^{\prime}\right\|_{0}^{2} \\
& \leq \tilde{E}^{\alpha}\left(\tilde{M}_{0}+C t P\left(\sup _{[0, t]} \tilde{E}\right)\right)
\end{aligned}
$$

with $\tilde{M}_{0}=P(\tilde{E}(0))$ and $P(\cdot)$ some polynomial function.

Proof. In the above energy estimate for Proposition 5.1, except for the term $I_{41}$ in (5.5), the others are the same as the proof of Proposition 5.1. Now we focus on the estimates of $I_{41}$. After detailed computations, $I_{41}$ is equal to the sum of the following terms:

$$
J:=\int_{0}^{t} \sum_{i=1}^{14} J_{i} d t, \quad \text { with } J_{i}=\int_{I} R\left(\eta^{\prime}\right) j_{i} \omega^{2+2 \mu} \partial_{t}^{5} v^{\prime} d x
$$

where the terms $j_{i}, i=1,2 \cdots, 14$ are the functions of $v^{\prime}, \partial_{t}^{k} v^{\prime}, k=1,2,3,4$ as in the following:

$$
\begin{aligned}
& j_{1}=v^{\prime}, \quad j_{2}=\partial_{t}^{4} v^{\prime}\left(v^{\prime}\right)^{2}, \quad j_{3}=\partial_{t}^{4} v^{\prime} \partial_{t} v^{\prime}, \quad j_{4}=\partial_{t}^{3} v^{\prime}\left(v^{\prime}\right)^{3}, \quad j_{5}=\partial_{t}^{3} v^{\prime} \partial_{t} v^{\prime} v^{\prime}, \\
& j_{6}=\partial_{t}^{3} v^{\prime} \partial_{t}^{2} v^{\prime}, \quad j_{7}=\partial_{t}^{2} v^{\prime}\left(v^{\prime}\right)^{4}, \quad j_{8}=\partial_{t}^{2} v^{\prime} \partial_{t} v^{\prime}\left(v^{\prime}\right)^{2}, \quad j_{9}=\partial_{t} v^{\prime}\left(v^{\prime}\right)^{5}, \quad j_{10}=\left(\partial_{t} v^{\prime}\right)^{3} v^{\prime}, \\
& j_{11}=\left(\partial_{t} v^{\prime}\right)^{2}\left(v^{\prime}\right)^{3}, \quad j_{12}=\left(\partial_{t} v^{\prime}\right)^{2} \partial_{t}^{2} v^{\prime}, \quad j_{13}=\left(v^{\prime}\right)^{7}, \quad j_{14}=\left(\partial_{t}^{2} v^{\prime}\right)^{2} v^{\prime} .
\end{aligned}
$$

For $J_{1}$, we have

$$
\left|J_{1}\right| \leq C|| \omega^{1+\mu} \partial_{t}^{5} v^{\prime}\left\|_{0}^{2}\right\| v^{\prime} \|_{\infty} \leq C P\left(\sup _{[0, t]} \tilde{E}\right)
$$

For $J_{2}, J_{3}, J_{4}$, we have

$$
\begin{aligned}
& \left|J_{2}\right| \leq C\left\|\omega^{1+\mu} \partial_{t}^{5} v^{\prime} \mid\right\|_{0}\left\|\omega^{1 / 2+\mu} \partial_{t}^{4} v^{\prime}\right\|\left\|_{0}\right\| v^{\prime}\left\|_{\infty}^{2}\right\| \omega \|_{\infty}^{1 / 2} \leq C P\left(\sup _{[0, t]} \tilde{E}\right) \\
& \left|J_{3}\right| \leq C\left\|\omega ^ { 1 + \mu } \partial _ { t } ^ { 5 } v ^ { \prime } \left|\left\|_{0}\right\| \omega^{1+\mu} \partial_{t}^{4} v^{\prime}\left\|\left.\right|_{0}\right\| v^{\prime}\left\|_{1 / 2}^{2}\right\| v^{\prime} \|_{1}^{1 / 2} \leq C P\left(\sup _{[0, t]} \tilde{E}\right)\right.\right. \\
& \left|J_{4}\right| \leq C\left\|\omega^{1+\mu} \partial_{t}^{5} v^{\prime} \mid\right\|_{0}\left\|\omega^{1+\mu} \partial_{t}^{3} v^{\prime}\right\|\left\|_{0}\right\| v^{\prime} \|_{\infty}^{3} \leq C P\left(\sup _{[0, t]} \tilde{E}\right)
\end{aligned}
$$

For $J_{5}$, we have

$$
\begin{aligned}
\left|J_{5}\right| & \leq\left. C\left\|\omega^{1+\mu} \partial_{t}^{5} v^{\prime}\right\|_{0}\left\|\omega^{1+\mu} \partial_{t}^{3} v^{\prime}\right\|_{L^{4}}\left\|\partial_{t} v^{\prime}\right\|\right|_{L^{4}}\left\|v^{\prime}\right\|_{\infty} \\
& \leq\left. C\left\|\omega^{1+\mu} \partial_{t}^{5} v^{\prime}\right\|\right|_{0}|| \omega^{1+\mu} \partial_{t}^{3} v^{\prime}\left\|_{1 / 2}\right\| \partial_{t} v^{\prime}\left\|_{1 / 2}\right\| v^{\prime} \|_{1} \\
& \leq \tilde{M}_{0}+C t P\left(\sup _{[0, t]} \tilde{E}\right)
\end{aligned}
$$


where we have used the similar method to (4.12) to deal with $\left\|\omega^{1+\mu} \partial_{t}^{3} v^{\prime}\right\|_{1 / 2}$. Similarly, we have

$$
\begin{aligned}
\left|J_{6}\right| & \leq C\left\|\omega^{1+\mu} \partial_{t}^{5} v^{\prime}\right\|_{0}|| \omega^{1+\mu} \partial_{t}^{3} v^{\prime}\left\|_{\infty}\right\| \partial_{t}^{2} v^{\prime} \|_{0} \\
& \leq\left.\left.\left\|\omega^{1+\mu} \partial_{t}^{5} v^{\prime}\right\|\right|_{0}\left\|\omega^{1+\mu} \partial_{t}^{3} v^{\prime}\right\|\right|_{1 / 2} ^{1 / 2}\left\|\omega^{1+\mu} \partial_{t}^{3} v^{\prime}\right\|_{0}^{1 / 2}\left\|\partial_{t}^{2} v^{\prime}\right\|_{0} \\
& \leq \tilde{M}_{0}+C t P\left(\sup _{[0, t]} \tilde{E}\right) \\
\left|J_{7}\right| & \leq C\left\|\omega^{1+\mu} \partial_{t}^{5} v^{\prime}\right\|_{0}|| \omega^{1+\mu} \partial_{t}^{2} v^{\prime}\left\|_{0}\right\| v^{\prime} \|_{\infty}^{4} \leq \tilde{M}_{0}+C t P\left(\sup _{[0, t]} \tilde{E}\right) \\
\left|J_{8}\right| & \leq C\left\|\omega^{1+\mu} \partial_{t}^{5} v^{\prime}\right\|_{0}|| \omega^{1+\mu} \partial_{t}^{2} v^{\prime}\left\|_{L^{4}}\right\| \partial_{t} v^{\prime}\left\|\left.\right|_{L^{4}}\right\| v^{\prime} \|_{\infty}^{2} \\
& \leq C\left\|\omega^{1+\mu} \partial_{t}^{5} v^{\prime}\right\|_{0}|| \omega^{1 / 2+\mu} \omega^{\prime} \partial_{t}^{2} v^{\prime}+\omega^{3 / 2+\mu} \partial_{t}^{2} v^{\prime \prime}\left\|_{0}\right\| \partial_{t} v^{\prime}\left\|_{1 / 2}\right\| v^{\prime} \|_{1}^{2} \\
& \leq \tilde{M}_{0}+C t P\left(\sup _{[0, t]} \tilde{E}\right)
\end{aligned}
$$

where we have used the fact

$$
\omega^{1+\mu} \partial_{t}^{2} v^{\prime}=\omega^{1+\mu} u_{2}^{\prime}+\int_{0}^{t} \omega^{1+\mu} \partial_{t}^{3} v^{\prime}
$$

and the similar method to (4.12) to deal with $\left\|\omega^{1+\mu} \partial_{t}^{2} v^{\prime}\right\|_{1 / 2}$.

Using (2.8), $J_{9}, J_{10}, J_{11}, J_{12}, J_{13}$ can be estimated as

$$
\begin{aligned}
& \left|J_{9}\right| \leq C|| \omega^{1+\mu} \partial_{t}^{5} v^{\prime}\left\|_{0}\right\| \partial_{t} v^{\prime}\left\|_{L^{4}}\right\| v^{\prime}\left\|_{L^{4}}\right\| v^{\prime} \|_{\infty}^{4} \leq \tilde{M}_{0}+C P\left(\sup _{[0, t]} \tilde{E}\right), \\
& \left|J_{10}\right| \leq C|| \omega^{1+\mu} \partial_{t}^{5} v^{\prime}\left\|_{0}\right\| \partial_{t} v^{\prime}\left\|_{L^{6}}^{3}\right\| v^{\prime} \|_{\infty}^{4} \leq \tilde{M}_{0}+C P\left(\sup _{[0, t]} \tilde{E}\right), \\
& \left|J_{11}\right| \leq C|| \omega^{1+\mu} \partial_{t}^{5} v^{\prime}\left\|_{0}\right\| \partial_{t} v^{\prime}\left\|_{L^{4}}^{2}\right\| v^{\prime} \|_{\infty}^{3} \leq \tilde{M}_{0}+C P\left(\sup _{[0, t]} \tilde{E}\right), \\
& \left|J_{12}\right| \leq C|| \omega^{1+\mu} \partial_{t}^{5} v^{\prime}\left\|_{0}\right\| \partial_{t} v^{\prime}\left\|_{L^{8}}^{2}\right\| \omega^{1+\mu} \partial_{t}^{2} v^{\prime} \|_{L^{4}} \leq \tilde{M}_{0}+C P\left(\sup _{[0, t]} \tilde{E}\right) \\
& \left|J_{13}\right| \leq C|| \omega^{1+\mu} \partial_{t}^{5} v^{\prime} \mid\left\|_{0}\right\| v^{\prime}\left\|_{0}\right\| v^{\prime} \|_{\infty}^{6} \leq C P\left(\sup _{[0, t]} \tilde{E}\right)
\end{aligned}
$$

For $J_{14}$, we have

$$
\begin{aligned}
\left|J_{14}\right| & \leq C\left\|\omega^{1+\mu} \partial_{t}^{5} v^{\prime}\right\|_{0}\left\|\omega^{1+\mu} \partial_{t}^{2} v^{\prime}\right\|_{\infty}\left\|v^{\prime}\right\|_{\infty}\left\|\partial_{t}^{2} v^{\prime}\right\|_{0} \\
& \leq C\left\|\omega^{1+\mu} \partial_{t}^{5} v^{\prime}\right\|_{0}\left\|\omega^{\mu} \omega^{\prime} \partial_{t}^{2} v^{\prime}+\omega^{1+\mu} \partial_{t}^{2} v^{\prime \prime}\right\|\left\|_{0}\right\| v^{\prime}\left\|_{\infty}\right\| \partial_{t}^{2} v^{\prime} \|_{0} \\
& \leq C P\left(\sup _{[0, t]} \tilde{E}\right) .
\end{aligned}
$$

Thus, using the same argument as Proposition 4.1, for $0<\alpha<1$, we obtain (5.8).

Similarly, we have 
Proposition 5.3. For $\gamma=\frac{3}{2}$, there exists a constant $\alpha \in(0,1)$, such that one has the following $\varepsilon-$ independent energy estimates:

$$
\begin{aligned}
&\left\|\omega^{1 / 2+\mu} \partial_{t}^{4} v\right\|_{0}^{2}+\left\|\omega^{1+\mu} \partial_{t}^{3} v^{\prime}\right\|_{0}^{2}+\left\|\omega^{1+\mu} \partial_{t}^{3} v\right\|_{0}^{2}+\varepsilon \int_{0}^{t}\left\|\omega^{1+\mu} \partial_{t}^{4} v^{\prime}\right\|_{0}^{2} \\
& \leq \tilde{E}^{\alpha}\left(\tilde{M}_{0}+C t P\left(\sup _{[0, t]} \tilde{E}\right)\right) \\
&\left\|\omega^{1 / 2+\mu} \partial_{t}^{2} v\right\|_{0}^{2}+\left\|\omega^{1+\mu} \partial_{t} v^{\prime}\right\|_{0}^{2}+\left\|\omega^{1+\mu} \partial_{t} v\right\|_{0}^{2}+\varepsilon \int_{0}^{t}\left\|\omega^{1+\mu} \partial_{t}^{2} v^{\prime}\right\|_{0}^{2} \\
& \leq \tilde{E}^{\alpha}\left(\tilde{M}_{0}+C t P\left(\sup _{[0, t]} \tilde{E}\right)\right) .
\end{aligned}
$$

\subsection{Estimates of higher-order spatial derivatives for $\gamma=\frac{3}{2}$}

Based on the energy estimate (5.8), by elliptic estimates we can derive the estimates of the higherorder spatial derivatives associated with the weights.

Proposition 5.4. For $\gamma=\frac{3}{2}$, there exists a constant $\alpha \in(0,1)$, such that one has the following estimates:

$$
\begin{aligned}
& \sup _{[0, t]}\left(\left.\left\|\omega^{1 / 2+\mu} \partial_{t}^{4} v^{\prime}\right\|\right|_{0} ^{2}+\left\|\omega^{3 / 2+\mu} \partial_{t}^{4} v^{\prime \prime}\right\|_{0}^{2}\right) \leq C\left(\tilde{E}^{\alpha}+1\right)\left(\tilde{M}_{0}+C t P\left(\sup _{[0, t]} \tilde{E}\right)\right) \\
& \sup _{[0, t]}\left(\left\|\omega^{1 / 2+\mu} \partial_{t}^{2} v^{\prime}\right\|_{0}^{2}+\left\|\omega^{1 / 2+\mu} \partial_{t}^{2} v^{\prime \prime}\right\|_{0}^{2}+\left\|\omega^{1 / 2+\mu} \partial_{t}^{2} v^{\prime \prime \prime}||_{0}^{2}+\right\| \omega^{1 / 2+\mu} v^{\prime}\left\|_{0}^{2}+\right\| \omega^{1 / 2+\mu} v^{\prime \prime} \|_{0}^{2}\right. \\
& \left.+\left\|\omega^{1 / 2+\mu} v^{\prime \prime \prime}\right\|_{0}^{2}+\left\|\omega^{3 / 2+\mu} \partial_{x}^{4} v\right\|_{0}^{2}\right) \leq C\left(\tilde{E}^{\alpha}+1\right)\left(\tilde{M}_{0}+C t P\left(\sup _{[0, t]} \tilde{E}\right)\right) .
\end{aligned}
$$

Proof. Choosing $k=4$ in (4.16), using the first term of (5.8), multiplying (4.16) by $\omega^{-1 / 2+\mu}$, then, using Lemma 2.1 and fundamental theorem of calculus, we obtain that, for any $t \in\left[0, T^{\varepsilon}\right]$,

$$
\begin{aligned}
\sup _{[0, t]}\left\|\omega^{-(1 / 2+\mu)}\left(\omega^{2+2 \mu} \partial_{t}^{4} v^{\prime} e^{S_{0}}\right)^{\prime}\right\|_{0} \leq C \sup _{[0, t]}\left\|\omega^{1 / 2+\mu} \partial_{t}^{6} v\right\|_{0} \\
+C \sup _{[0, t]} \sum_{\alpha=1}^{4}\left\|\omega^{-(1 / 2+\mu)}\left(\omega^{2+2 \mu} \partial_{t}^{\alpha} \frac{1}{\left(\eta^{\prime}\right)^{\gamma+1}} \partial_{t}^{4-\alpha} v^{\prime} e^{S_{0}}\right)^{\prime}\right\|_{0} \\
+C \sup _{[0, t]}\left\|\omega^{-(1 / 2+\mu)}\left[\left(1-\frac{1}{\left(\eta^{\prime}\right)^{\gamma+1}}\right)\left(\omega^{2+2 \mu} \partial_{t}^{4} v^{\prime} e^{S_{0}}\right)^{\prime}\right]\right\|_{0} \\
+C \sup _{[0, t]}\left\|\frac{\omega^{3 / 2+\mu} \partial_{t}^{4} v^{\prime} e_{0}^{S} \eta^{\prime \prime}}{\eta^{\prime(\gamma+2)}}\right\|_{0}=: \sum_{i=1}^{4} I_{i} .
\end{aligned}
$$


Now we estimate each term on the right-hand side of (5.13). First, we use the estimate (5.8) to obtain, for each $t \in\left[0, T_{k}\right]$,

$$
I_{1}=\sup _{[0, t]}\left\|\omega^{1 / 2+\mu} \partial_{t}^{6} v\right\|_{0} \leq \tilde{E}^{\alpha / 2}\left(\tilde{M}_{0}+C t P\left(\sup _{[0, t]} \tilde{E}\right)\right) .
$$

The remaining terms will be estimated by using the definition of the energy function $\tilde{E}$. For the second term, from (4.31), after detailed computations, we have

$$
\begin{aligned}
I_{2} & =\sum_{\alpha=1}^{4}\left\|\omega^{-(1 / 2+\mu)}\left(\omega^{2+2 \mu} \partial_{t}^{\alpha} \frac{1}{\left(\eta^{\prime}\right)^{\gamma+1}} \partial_{t}^{4-\alpha} v^{\prime} e_{0}^{S}\right)^{\prime}\right\|_{0} \\
& =\sum_{\alpha=1}^{4}\left\|-\frac{1}{\gamma+1} \omega^{-(1 / 2+\mu)}\left(\omega^{2+2 \mu} \partial_{t}^{4-\alpha} v^{\prime} e_{0}^{S} \int_{0}^{t} \partial_{t}^{\alpha}\left(\frac{v^{\prime}}{\left(\eta^{\prime}\right)^{\gamma+2}}\right)\right)^{\prime}\right\|_{0} \leq \sum_{i=1}^{74} K_{i},
\end{aligned}
$$

where we have used the fact $1 / 2 \leq \eta^{\prime} \leq 3 / 2$ and $\underline{S} \leq S_{0}^{\prime} \leq \bar{S}$.

For $i=1,2, \cdots, 17, K_{i}=C\left\|\left(\omega^{1 / 2+\mu}+\omega^{3 / 2+\mu}\right) k_{i}\right\|_{0}$ with

$$
\begin{aligned}
& k_{1}=\partial_{t}^{3} v^{\prime} \int_{0}^{t}\left(v^{\prime}\right)^{2}, k_{2}=\partial_{t}^{3} v^{\prime} \int_{0}^{t} \partial_{t} v^{\prime}, k_{3}=\partial_{t}^{2} v^{\prime} \int_{0}^{t}\left(v^{\prime}\right)^{3}, k_{4}=\partial_{t}^{2} v^{\prime} \int_{0}^{t} v^{\prime} \partial_{t} v^{\prime}, \\
& k_{5}=\partial_{t}^{2} v^{\prime} \int_{0}^{t} \partial_{t}^{2} v^{\prime}, k_{6}=\partial_{t} v^{\prime} \int_{0}^{t}\left(v^{\prime}\right)^{4}, k_{7}=\partial_{t} v^{\prime} \int_{0}^{t}\left(v^{\prime}\right)^{2} \partial_{t} v^{\prime}, k_{8}=\partial_{t} v^{\prime} \int_{0}^{t}\left(\partial_{t} v^{\prime}\right)^{2}, \\
& k_{9}=\partial_{t} v^{\prime} \int_{0}^{t} v^{\prime} \partial_{t}^{2} v^{\prime}, k_{10}=\partial_{t} v^{\prime} \int_{0}^{t} \partial_{t}^{3} v^{\prime}, k_{11}=v^{\prime} \int_{0}^{t}\left(v^{\prime}\right)^{5}, k_{12}=v^{\prime} \int_{0}^{t}\left(v^{\prime}\right)^{3} \partial_{t} v^{\prime}, \\
& k_{13}=v^{\prime} \int_{0}^{t}\left(v^{\prime}\right)^{2} \partial_{t}^{2} v^{\prime}, k_{14}=v^{\prime} \int_{0}^{t} v^{\prime}\left(\partial_{t} v^{\prime}\right)^{2}, k_{15}=v^{\prime} \int_{0}^{t} \partial_{t} v^{\prime} \partial_{t}^{2} v^{\prime}, k_{16}=v^{\prime} \int_{0}^{t} v^{\prime} \partial_{t}^{3} v^{\prime}, \\
& k_{17}=v^{\prime} \int_{0}^{t} \partial_{t}^{4} v^{\prime} .
\end{aligned}
$$

For $i=18,19, \cdots, 58, K_{i}=C\left\|\omega^{3 / 2+\mu} k_{i}\right\|_{0}$ with

$$
\begin{aligned}
& k_{18}=\partial_{t}^{3} v^{\prime} \int_{0}^{t} v^{\prime} v^{\prime \prime}, k_{19}=\partial_{t}^{3} v^{\prime} \int_{0}^{t} \partial_{t} v^{\prime \prime}, k_{20}=\partial_{t}^{2} v^{\prime} \int_{0}^{t}\left(v^{\prime}\right)^{2} v^{\prime \prime}, k_{21}=\partial_{t}^{2} v^{\prime} \int_{0}^{t} v^{\prime \prime} \partial_{t} v^{\prime}, \\
& k_{22}=\partial_{t}^{2} v^{\prime} \int_{0}^{t} v^{\prime} \partial_{t} v^{\prime \prime}, k_{23}=\partial_{t}^{2} v^{\prime} \int_{0}^{t} \partial_{t}^{2} v^{\prime \prime}, k_{24}=\partial_{t} v^{\prime} \int_{0}^{t}\left(v^{\prime}\right)^{3} v^{\prime \prime}, k_{25}=\partial_{t} v^{\prime} \int_{0}^{t} v^{\prime} \partial_{t} v^{\prime}, \\
& k_{26}=\partial_{t} v^{\prime} \int_{0}^{t}\left(v^{\prime}\right)^{2} \partial_{t} v^{\prime \prime}, k_{27}=\partial_{t} v^{\prime} \int_{0}^{t} \partial_{t} v^{\prime} \partial_{t} v^{\prime \prime}, k_{28}=\partial_{t} v^{\prime} \int_{0}^{t} \partial_{t}^{2} v^{\prime} v^{\prime \prime}, k_{29}=\partial_{t} v^{\prime} \int_{0}^{t} v^{\prime} \partial_{t}^{2} v^{\prime \prime}, \\
& k_{30}=\partial_{t} v^{\prime} \int_{0}^{t} \partial_{t}^{3} v^{\prime \prime}, k_{31}=v^{\prime} \int_{0}^{t}\left(v^{\prime}\right)^{4} v^{\prime \prime}, k_{32}=v^{\prime} \int_{0}^{t}\left(v^{\prime}\right)^{2} v^{\prime \prime} \partial_{t} v^{\prime}, k_{33}=v^{\prime} \int_{0}^{t}\left(v^{\prime}\right)^{3} \partial_{t} v^{\prime \prime}, \\
& k_{34}=v^{\prime} \int_{0}^{t} v^{\prime \prime} \partial_{t} v^{\prime}, k_{35}=v^{\prime} \int_{0}^{t} \partial_{t} v^{\prime} \partial_{t} v^{\prime \prime}, k_{36}=v^{\prime} \int_{0}^{t} \partial_{t}^{2} v^{\prime \prime}, k_{37}=v^{\prime} \int_{0}^{t} v^{\prime} v^{\prime \prime} \partial_{t}^{2} v^{\prime}, \\
& k_{38}=v^{\prime} \int_{0}^{t} \partial_{t}^{2} v^{\prime} \partial_{t} v^{\prime \prime}, k_{39}=v^{\prime} \int_{0}^{t} \partial_{t} v^{\prime} \partial_{t}^{2} v^{\prime \prime}, k_{40}=v^{\prime} \int_{0}^{t} v^{\prime} \partial_{t}^{3} v^{\prime \prime}, k_{41}=v^{\prime} \int_{0}^{t} \partial_{t}^{3} v^{\prime} v^{\prime \prime}, \\
& k_{42}=v^{\prime} \int_{0}^{t} \partial_{t}^{4} v^{\prime \prime}, k_{43}=\partial_{t}^{3} v^{\prime \prime} \int_{0}^{t}\left(v^{\prime}\right)^{2}, k_{44}=\partial_{t}^{3} v^{\prime \prime} \int_{0}^{t} \partial_{t} v^{\prime}, k_{45}=\partial_{t}^{2} v^{\prime \prime} \int_{0}^{t}\left(v^{\prime}\right)^{3},
\end{aligned}
$$




$$
\begin{aligned}
& k_{46}=\partial_{t}^{2} v^{\prime \prime} \int_{0}^{t} v^{\prime \prime} \partial_{t} v^{\prime}, k_{47}=\partial_{t}^{2} v^{\prime \prime} \int_{0}^{t} \partial_{t}^{2} v^{\prime}, k_{48}=\partial_{t} v^{\prime \prime} \int_{0}^{t}\left(v^{\prime}\right)^{4}, k_{49}=\partial_{t} v^{\prime \prime} \int_{0}^{t}\left(v^{\prime}\right)^{2} \partial_{t} v^{\prime}, \\
& k_{50}=\partial_{t} v^{\prime \prime} \int_{0}^{t}\left(\partial_{t} v^{\prime}\right)^{2}, k_{51}=\partial_{t} v^{\prime \prime} \int_{0}^{t} v^{\prime} \partial_{t}^{2} v^{\prime}, k_{52}=\partial_{t} v^{\prime \prime} \int_{0}^{t} \partial_{t}^{3} v^{\prime}, k_{53}=v^{\prime \prime} \int_{0}^{t}\left(v^{\prime}\right)^{2}\left(\partial_{t} v^{\prime}\right)^{2}, \\
& k_{54}=v^{\prime \prime} \int_{0}^{t}\left(v^{\prime}\right)^{2} \partial_{t}^{2} v^{\prime}, k_{55}=v^{\prime \prime} \int_{0}^{t} \partial_{t} v^{\prime} \partial_{t}^{2} v^{\prime}, k_{56}=v^{\prime \prime} \int_{0}^{t} v^{\prime} \partial_{t}^{3} v^{\prime}, k_{57}=v^{\prime \prime} \int_{0}^{t} \partial_{t}^{4} v^{\prime} .
\end{aligned}
$$

For $i=58,59, \cdots, 74, K_{i}=C\left\|\omega^{3 / 2+\mu} k_{i}\right\|_{0}$, where each $k_{i}$ has the form similar to the term $k_{i-17}$ in (5.16) but every integrand function has an additional term $\eta^{\prime \prime}$, for example,

$$
k_{58}=\partial_{t}^{3} v^{\prime} \int_{0}^{t}\left(v^{\prime}\right)^{2} \eta^{\prime \prime}, k_{59}=\partial_{t}^{3} v^{\prime} \int_{0}^{t} \partial_{t} v^{\prime \prime} \eta^{\prime \prime}, \cdots, k_{74}=v^{\prime} \int_{0}^{t} \partial_{t}^{4} v^{\prime} \eta^{\prime \prime} .
$$

Before deriving the estimates of $K_{i}, i=1,2, \cdots, 74$, similarly to (4.24), one has

$$
\begin{gathered}
\sum_{j=1}^{3}\left\{\left\|\omega^{3 / 2+\mu} \partial_{t}^{5-2 j} \partial_{x}^{j+1} v\right\|_{0}^{2}+\sum_{i=1}^{j}\left\|\omega^{1 / 2+\mu} \partial_{t}^{5-2 j} \partial_{x}^{i} v\right\|_{0}^{2}+\sum_{i=-1}^{j}\left\|\omega^{1+\mu} \partial_{t}^{4-2 j} \partial_{x}^{i+1} v\right\|_{0}^{2}\right\} \\
\leq \tilde{M}_{0}+C t P\left(\sup _{[0, t]} \tilde{E}\right) .
\end{gathered}
$$

For $K_{1}, K_{2}, K_{4}, K_{5}$, using the estimates (5.17), we have

$$
\begin{aligned}
K_{1} & =C\left\|\left(\omega^{1 / 2+\mu}+\omega^{3 / 2+\mu}\right) \partial_{t}^{3} v^{\prime} \int_{0}^{t}\left(v^{\prime}\right)^{2}\right\|_{0} \leq C\left\|\omega^{1 / 2+\mu} \partial_{t}^{3} v^{\prime}\right\|_{0} \int_{0}^{t}\left\|v^{\prime}\right\|_{\infty}^{2} \\
& \leq \tilde{M}_{0}+C t P\left(\sup _{[0, t]} \tilde{E}\right), \\
K_{2} & =C\left\|\left(\omega^{1 / 2+\mu}+\omega^{3 / 2+\mu}\right) \partial_{t}^{3} v^{\prime} \int_{0}^{t} \partial_{t} v^{\prime}\right\|_{0} \leq C\left\|\omega^{1 / 2+\mu} \partial_{t}^{3} v^{\prime}\right\|_{0}\left(\left\|v^{\prime}\right\|_{\infty}+\left\|u_{0}^{\prime}\right\|_{\infty}\right) \\
& \leq C \tilde{E}^{1 / 2}\left(\tilde{M}_{0}+\tilde{E}^{1 / 2}\right), \\
K_{4} & =C\left\|\left(\omega^{1 / 2+\mu}+\omega^{3 / 2+\mu}\right) \partial_{t}^{2} v^{\prime} \int_{0}^{t} v^{\prime} \partial_{t} v^{\prime}\right\|_{0} \leq C\left\|\omega^{1 / 2+\mu} \partial_{t}^{2} v^{\prime}\right\|_{L^{4}}\left\|\partial_{t} v^{\prime}\right\|_{L^{4}} \int_{0}^{t}\left\|v^{\prime}\right\|_{\infty} \\
& \leq \tilde{M}_{0}+C t P\left(\sup _{[0, t]} \tilde{E}\right), \\
K_{5} & =C\left\|\left(\omega^{1 / 2+\mu}+\omega^{3 / 2+\mu}\right) \partial_{t}^{2} v^{\prime} \int_{0}^{t} \partial_{t}^{2} v^{\prime}\right\|_{0} \leq C\left\|\omega^{1 / 2+\mu} \partial_{t}^{2} v^{\prime}\right\|_{\infty} \int_{0}^{t}\left\|\partial_{t}^{2} v^{\prime}\right\|_{0} \\
& \leq C t P\left(\sup _{[0, t]} \tilde{E}\right),
\end{aligned}
$$


For $K_{7}, K_{8}, K_{9}$, we have

$$
\begin{aligned}
K_{7} & =C\left\|\left(\omega^{1 / 2+\mu}+\omega^{3 / 2+\mu}\right) \partial_{t} v^{\prime} \int_{0}^{t}\left(v^{\prime}\right)^{2} \partial_{t} v^{\prime}\right\|_{0} \leq C \int_{0}^{t}\left\|\omega^{1 / 2+\mu} \partial_{t} v^{\prime}\right\|\left\|_{L^{8}}\right\| \partial_{t} v^{\prime}\left\|L_{L^{8}}\right\| v^{\prime} \|_{L^{4}}^{2} \\
& \leq C t P\left(\sup _{[0, t]} \tilde{E}\right), \\
K_{8} & =C\left\|\left(\omega^{1 / 2+\mu}+\omega^{3 / 2+\mu}\right) \partial_{t} v^{\prime} \int_{0}^{t}\left(\partial_{t} v^{\prime}\right)^{2}\right\|_{0} \leq C \int_{0}^{t}\left\|\omega^{1 / 2+\mu} \partial_{t} v^{\prime}\right\|_{L^{4}}\left\|\partial_{t} v^{\prime}\right\|_{L^{8}}^{2} \\
& \leq C t P\left(\sup _{[0, t]} \tilde{E}\right), \\
K_{9} & =\left\|\left(\omega^{1 / 2+\mu}+\omega^{3 / 2+\mu}\right) \partial_{t} v^{\prime} \int_{0}^{t} \partial_{t}^{2} v^{\prime} v^{\prime}\right\|_{0} \leq C \int_{0}^{t}\left\|\omega^{1 / 2+\mu} \partial_{t} v^{\prime}\right\|_{\infty}\left\|\partial_{t}^{2} v^{\prime}\right\|\left\|_{0}\right\| v^{\prime} \|_{\infty} \\
& \leq C t P\left(\sup _{[0, t]} \tilde{E}\right) .
\end{aligned}
$$

For $K_{10}$, using the same method as dealing with $I_{78}$ in (4.27) and (4.29), we have

$$
\begin{aligned}
K_{10} & =\left\|\left(\omega^{1 / 2+\mu}+\omega^{3 / 2+\mu}\right) \partial_{t} v^{\prime} \int_{0}^{t} \partial_{t}^{3} v^{\prime}\right\|_{0} \leq C\left\|\omega^{1 / 2+\mu} \partial_{t}^{2} v^{\prime}\right\|_{\infty}\left\|\partial_{t} v^{\prime}\right\|_{0} \\
& \leq C\left\|\omega^{1 / 2+\mu} \partial_{t}^{2} v^{\prime}\right\|_{3 / 4}\left\|\partial_{t} v^{\prime}\right\|_{0} \leq C\left\|\omega^{1 / 2+\mu} \partial_{t}^{2} v^{\prime}\right\|\left\|_{0}^{1-\alpha}\right\|\left(\omega^{1 / 2+\mu} \partial_{t}^{2} v^{\prime}\right)^{\prime}\left\|L_{L^{r}}^{\alpha}\right\| \partial_{t} v^{\prime} \|_{0} \\
& \leq C\left\|\omega^{1 / 2+\mu} \partial_{t}^{2} v^{\prime}\right\|_{0}^{1-\alpha}\left(\left\|\omega^{\mu-1 / 2} \partial_{t}^{2} v^{\prime}\right\|_{L^{r}}^{\alpha}+\left\|\omega^{1 / 2+\mu} \partial_{t}^{2} v^{\prime \prime}\right\|_{L^{r}}^{\alpha}\right)\left\|\partial_{t} v^{\prime}\right\|_{0} \\
& \leq C\left\|\omega^{1 / 2+\mu} \partial_{t}^{2} v^{\prime}\right\|_{0}^{1-\alpha}\left(\left\|\omega^{\mu}\right\|_{\infty}\left\|\frac{\partial_{t}^{2} v^{\prime}}{\sqrt{\omega}}\right\|_{L^{r}}^{\alpha}+\left\|\omega^{1 / 2+\mu} \partial_{t}^{2} v^{\prime \prime}\right\|_{L^{r}}^{\alpha}\right)\left\|\partial_{t} v^{\prime}\right\|_{0} \\
& \leq C\left\|\omega^{1 / 2+\mu} \partial_{t}^{2} v^{\prime}\right\|_{0}^{1-\alpha}\left(\left\|\frac{1}{\sqrt{\omega}}\right\|_{L^{\beta}}^{\alpha}\left\|\partial_{t}^{2} v^{\prime}\right\|_{0}^{\alpha}+\left\|\omega^{1 / 2+\mu} \partial_{t}^{2} v^{\prime \prime}\right\|_{L^{r}}^{\alpha}\right)\left\|\partial_{t} v^{\prime}\right\|_{0} \\
& \leq C \tilde{E}^{\alpha / 2} .
\end{aligned}
$$

To ensure $\frac{1}{2} \beta<1$, here we choose $\frac{1}{\beta} \in\left(\frac{1}{2}, \frac{1}{r}\right)$, and $\alpha_{0}<\alpha<1$ with $0 \leq \alpha_{0}=\frac{3}{4(1+\mu)}<\frac{3}{4}$ as $1<\gamma<2$ and $\frac{1}{r}=\frac{3}{2}-\frac{3}{4 \alpha}$. Similarly,

$$
\begin{aligned}
K_{19} & =\left\|\omega^{3 / 2+\mu} \partial_{t}^{3} v^{\prime} \int_{0}^{t} \partial_{t} v^{\prime \prime}\right\|_{0} \leq C\left(\left\|\omega^{1 / 2+\mu} v^{\prime \prime}\right\|_{\infty}+\left\|\omega^{1 / 2+\mu} u_{0}^{\prime \prime}\right\|_{\infty}\right)\left\|\omega^{1 / 2+\mu} \partial_{t}^{3} v^{\prime}\right\|_{0} \\
& \leq C \tilde{E}^{\alpha / 2} \\
K_{52} & =\left\|\omega^{3 / 2+\mu} \partial_{t} v^{\prime \prime} \int_{0}^{t} \partial_{t}^{3} v^{\prime}\right\|_{0} \leq C\left\|\omega^{1 / 2+\mu} \int_{0}^{t} \partial_{t}^{3} v^{\prime}\right\|_{\infty}\left\|\omega^{1 / 2+\mu} \partial_{t} v^{\prime \prime}\right\|_{0} \leq C \tilde{E}^{\alpha / 2} .
\end{aligned}
$$

For $K_{18}$, we have

$$
K_{18}=\left\|\omega^{3 / 2+\mu} \partial_{t}^{3} v^{\prime} \int_{0}^{t} v^{\prime} v^{\prime \prime}\right\|_{0} \leq C \int_{0}^{t}\left\|\omega^{3 / 2+\mu} \partial_{t}^{3} v^{\prime \prime}\right\|_{\infty}\left\|v^{\prime \prime}\right\|\left\|_{0}\right\| v^{\prime} \|_{\infty} \leq \tilde{M}_{0}+C t P\left(\sup _{[0, t]} \tilde{E}\right) .
$$

For $K_{27}$, we have

$$
K_{27}=\left\|\omega^{3 / 2+\mu} \partial_{t} v^{\prime} \int_{0}^{t} \partial_{t} v^{\prime} \partial_{t} v^{\prime \prime}\right\|_{0} \leq C \int_{0}^{t}\left\|\omega^{3 / 2+\mu} \partial_{t} v^{\prime \prime}\right\|_{\infty}\left\|\partial_{t} v^{\prime}\right\|_{L^{4}}^{2} \leq \tilde{M}_{0}+C t P(\underset{[0, t]}{\sup } \tilde{E}) .
$$


By detailed analysis, we find that the estimates of $K_{i}, i=3,6,11,12,13,16,17,31,33,36,40,42,43$, $44,45,48,51,57$ are the same as $K_{1}$; the estimates of $K_{i}, i=14,15,29,50$ are the same as $K_{4}$; the estimates of $K_{i}, i=23,39$ are the same as $K_{5}$; the estimates of $K_{i}, i=53,54,55,56$ are the same as $K_{9}$; the estimates of $K_{i}, i=20,21,22,24,28,32,34,37,38,41,46,47,49$ are the same as $K_{18}$, thus we omit them. Due to $\eta^{\prime \prime}=\int_{0}^{t} v^{\prime \prime}$, similarly to $K_{18}$, we also have

$$
\begin{aligned}
K_{58} & =C\left\|\omega^{3 / 2+\mu} \partial_{t}^{3} v^{\prime} \int_{0}^{t}\left(v^{\prime}\right)^{2} \eta^{\prime \prime}\right\|_{0} \leq \int_{0}^{t}\left\|v^{\prime}\right\|_{\infty}^{2}\left\|\omega^{3 / 2+\mu} \partial_{t}^{3} v^{\prime}\right\|_{\infty}\left\|\int_{0}^{t} v^{\prime \prime}\right\|_{0} \\
& \leq \tilde{M}_{0}+C t P\left(\sup _{[0, t]} \tilde{E}\right),
\end{aligned}
$$

and the estimates of $K_{i}, i=59, \cdots, 74$, can also be obtained in the same way. Substituting all the estimates of $K_{i}$ into (5.15), one has

$$
I_{2} \leq \tilde{E}^{\alpha / 2}\left(\tilde{M}_{0}+C t P\left(\sup _{[0, t]} \tilde{E}\right)\right)+C t P\left(\sup _{[0, t]} \tilde{E}\right) .
$$

From (4.31), the third term $I_{3}$ on the right-hand side of (5.13) can be controlled by

$$
I_{3}=\left\|\omega\left[\left(1-\frac{1}{\left(\eta^{\prime}\right)^{\gamma+1}}\right)\left(\omega^{2+2 \mu} \partial_{t}^{4} v^{\prime} e_{0}^{S}\right)^{\prime}\right]\right\|_{0} \leq C t P\left(\sup _{[0, t]} \tilde{E}\right) .
$$

The last term of (5.13) can be estimated as

$$
I_{4} \leq C\left\|\omega^{2+\mu} \partial_{t}^{4} v^{\prime}\right\|_{\infty}\left\|\int_{0}^{t} v^{\prime \prime}\right\|_{0} \leq C t P\left(\sup _{[0, t]} \tilde{E}\right) .
$$

Substituting (5.14), (5.20) and (5.21) into (5.13), we have

$$
\left\|\omega^{-(1 / 2+\mu)}\left(\omega^{2+2 \mu} \partial_{t}^{4} v^{\prime} e_{0}^{S}\right)^{\prime}\right\|_{0}^{2} \leq C\left(\tilde{E}^{\alpha}+1\right)\left(\tilde{M}_{0}+t P\left(\sup _{[0, t]} \tilde{E}\right)\right)+C t P\left(\sup _{[0, t]} \tilde{E}\right) .
$$

Similarly to (4.33)-(4.34), we have

$$
\begin{aligned}
& \sup _{[0, t]}\left(\left\|\omega^{1 / 2+\mu} \partial_{t}^{4} v^{\prime}\right\|_{0}^{2}+\left\|\omega^{3 / 2+\mu} \partial_{t}^{4} v^{\prime \prime}\right\|_{0}^{2}\right) \\
& \leq C\left(1+\tilde{E}^{\alpha}\right)\left(\tilde{M}_{0}+t P\left(\sup _{[0, t]} \tilde{E}\right)\right)+C t P\left(\sup _{[0, t]} \tilde{E}\right) .
\end{aligned}
$$

Choosing the multipliers $\omega^{-(1 / 2+\mu)}$, and replacing the first term $\omega^{1 / 2+\mu} \partial_{t}^{6} v$ on the right hand-side of (5.13) by $\omega^{1 / 2+\mu} \partial_{t}^{4} v, \omega^{1 / 2+\mu} \partial_{t}^{4} v^{\prime}, \omega^{1 / 2+\mu} \partial_{t}^{2} v, \omega^{1 / 2+\mu} \partial_{t}^{2} v^{\prime}, \omega^{1 / 2+\mu} \partial_{t}^{2} v^{\prime \prime}$, respectively, we can obtain the estimates (5.12).

Furthermore, Choosing the multiplier $\omega^{-\mu}$, and replacing $\omega^{1 / 2+\mu} \partial_{t}^{k+2} v$ in (5.13) by $\omega^{1+\mu} \partial_{t}^{5} v$, $\omega^{1+\mu} \partial_{t}^{5} v^{\prime}, \omega^{1+\mu} \partial_{t}^{3} v^{\prime}, \omega^{1+\mu} \partial_{t}^{3} v^{\prime \prime}$, respectively, we have the following proposition: 
Proposition 5.5. For $\gamma=\frac{3}{2}$, there exists a constant $\alpha \in(0,1)$, such that one has the following estimate:

$$
\begin{gathered}
\sup _{[0, t]}\left(\left.\left\|\omega^{1+\mu} \partial_{t}^{3} v^{\prime}\right\|\right|_{0} ^{2}+\left\|\omega^{1+\mu} \partial_{t}^{3} v^{\prime \prime}\right\|_{0}^{2}+\left\|\omega^{2+\mu} \partial_{t}^{3} v^{\prime \prime \prime}||_{0}^{2}+\right\| \omega^{1+\mu} \partial_{t} v^{\prime \prime}\left\|_{0}^{2}+\right\| \omega^{1+\mu} \partial_{t} v^{\prime \prime \prime} \|_{0}^{2}\right. \\
\left.+\left\|\omega^{2+\mu} \partial_{t} \partial_{x}^{4} v\right\|_{0}^{2}\right) \leq C\left(\tilde{E}^{\alpha}+1\right)\left(\tilde{M}_{0}+C t P\left(\sup _{[0, t]} \tilde{E}\right)\right)
\end{gathered}
$$

\section{The proof of Theorem 2.1}

We are now ready to finish the proof of Theorem 2.1 as follows. From (2.18), the Propositions 4.1 4.4 for $2 \leq \gamma<3$ and Propositions 5.2 5.4 for $\gamma=\frac{3}{2}$, one has

$$
\sup _{[0, T]} E(t) \leq M_{0}+C t P\left(\sup _{[0, t]} E\right)+C \sup _{[0, t]} E^{\alpha}\left(M_{0}+t P\left(\sup _{[0, t]} E\right)\right)
$$

where $M_{0}=P(E(0)), P(\cdot)$ is some polynomial function, and $0<\alpha<1$. By Young's inequality and adjusting the constants, one gets

$$
\sup _{[0, t]} E(t) \leq M_{0}+C t P\left(\sup _{[0, t]} E\right)
$$

which yields as in [5] a time of existence $T_{1}$ independent of $\varepsilon$, as well as an energy estimate on the time interval $\left(0, T_{1}\right)$ independent of $\varepsilon$ of the form:

$$
\sup _{[0, t]} E(t) \leq 2 M_{0}
$$

By the $\epsilon$-independent estimate (6.1), there exists a subsequence of $v^{\epsilon}$ converging to $v$ in $L^{2}\left(0, T ; H^{2}(I)\right)$ with $\eta=x+\int_{0}^{t} v(x, s) d s$. The standard compactness arguments shows that $v$ is a solution to (2.5). Thus, we can prove Theorem 2.1 for $2 \leq \gamma<3$ and $\gamma=\frac{3}{2}$. Moreover, in the above process, we find that the method can be extended to all the cases of $1<\gamma<2$, thus Theorem 2.1 can be proved for all the general case $1<\gamma<3$.

Now, we are ready to prove the uniqueness of solutions. For two solutions $v_{1}(x, t), v_{2}(x, t)$ satisfying Theorem 2.1 to the free-boundary problem of the compressible Euler equations (1.1), we want to prove $v_{1}(x, t)=v_{2}(x, t)$. In fact, from (2.20), there exits three positive constants $c_{1}, c_{2}, c_{3}$ such that

$$
c_{1} \leq \partial_{x} \eta_{i}(x, t) \leq c_{2}, \quad \text { and }\left|\partial_{x} v_{i}(x, t)\right| \leq c_{3}, \quad(x, t) \in[0,1] \times[0, T], \quad i=1,2
$$

We define $\delta v=v_{1}-v_{2}$, then $\delta v$ satisfy the following equation:

$$
\omega^{1+2 \mu} \partial_{t} \delta v+\left[\omega^{2+2 \mu} e^{S_{0}}\left(\frac{1}{\left(\eta_{1}^{\prime}\right)^{\gamma}}-\frac{1}{\left(\eta_{2}^{\prime}\right)^{\gamma}}\right)\right]^{\prime}=0
$$


By considering the fifth differential version of (6.3), from the Proposition 5.1, we have the following equation for $\delta v$ :

$$
\begin{aligned}
\frac{1}{2} \int_{I} \omega^{1+2 \mu}\left|\partial_{t}^{5} \delta v\right|^{2}+\frac{\gamma}{2} \int_{I}\left|\partial_{t}^{4} \delta v^{\prime}\right|^{2} \frac{\omega^{2+2 \mu}}{\left(\eta_{1}^{\prime}\right)^{\gamma+1}} e^{S_{0}}+\epsilon \int_{I} \omega^{2+2 \mu}\left|\partial_{t}^{5} \delta v^{\prime}\right|^{2} e^{S_{0}} \\
=-\frac{(\gamma+1) \gamma}{2} \int_{0}^{t} \int_{I} \omega^{2+2 \mu} e^{S_{0}}\left(\frac{1}{\left(\eta_{1}^{\prime}\right)^{\gamma+2}} v_{1}^{\prime}\left|\partial_{t}^{4} v_{1}^{\prime}\right|^{2}-\frac{1}{\left(\eta_{2}^{\prime}\right)^{\gamma+2}} v_{2}^{\prime}\left|\partial_{t}^{4} v_{2}^{\prime}\right|^{2}\right) \\
\quad-\sum_{\alpha=1}^{4} c_{\alpha} \int_{0}^{t} \int_{I} \omega^{2+2 \mu} e^{S_{0}}\left(\partial_{t}^{\alpha} \frac{1}{\left(\eta_{1}^{\prime}\right)^{\gamma+1}} \partial_{t}^{4-\alpha} v_{1}^{\prime} \partial_{t}^{5} v_{1}^{\prime}-\partial_{t}^{\alpha} \frac{1}{\left(\eta_{2}^{\prime}\right)^{\gamma+1}} \partial_{t}^{4-\alpha} v_{2}^{\prime} \partial_{t}^{5} v_{2}^{\prime}\right) \\
\quad-\gamma \int_{I} \omega^{2+2 \mu} e^{S_{0}}\left(\frac{\partial_{t}^{4} v_{1}^{\prime} \partial_{t}^{4} v_{2}^{\prime}}{\left(\eta_{1}^{\prime}\right)^{\gamma+1}}+\frac{1}{2}\left(\frac{1}{\left(\eta_{1}^{\prime}\right)^{\gamma+1}}+\frac{1}{\left.\left.\left(\eta_{2}^{\prime}\right)^{\gamma+1}\right)\left|\partial_{t}^{4} v_{2}^{\prime}\right|^{2}\right)}\right.\right. \\
\quad+2 \epsilon \int_{I} \omega^{2+2 \mu} \partial_{t}^{5} v_{1}^{\prime} \partial_{t}^{5} v_{2}^{\prime} e^{S_{0}} .
\end{aligned}
$$

Using the energy estimate and weighted embedding inequality (2.13), we obtain the analogous version of Proposition 5.1 and Proposition 4.2 for $\delta v$, and $\partial_{t}^{4} \delta v \in L^{\infty}\left(0, T ; L^{2}(I)\right)$. Next, we consider the elliptic estimates for higher-order spatial derivatives, $\delta v$ satisfies the following equation

$$
\left(\omega^{2+2 \mu} \partial_{t}^{k} \delta v^{\prime} e^{S_{0}}\right)^{\prime}-\frac{\varepsilon}{\gamma} \partial_{t}\left(\omega^{2+2 \mu} \partial_{t}^{k} \delta v^{\prime} e^{S_{0}}\right)^{\prime}=\delta g
$$

where

$$
\begin{aligned}
\delta g= & -\frac{1}{\gamma} \omega^{1+2 \mu} \partial_{t}^{k+2} \delta v+\sum_{\alpha=1}^{k}\left(c_{\alpha} \omega^{2+2 \mu} e^{S_{0}}\left(\partial_{t}^{\alpha} \frac{1}{\left(\eta_{1}^{\prime}\right)^{\gamma+1}} \partial_{t}^{k-\alpha} v_{1}^{\prime}-\partial_{t}^{\alpha} \frac{1}{\left(\eta_{2}^{\prime}\right)^{\gamma+1}} \partial_{t}^{k-\alpha} v_{2}^{\prime}\right)\right)^{\prime} \\
& +\left[\left(1-\frac{1}{\left(\eta_{1}^{\prime}\right)^{\gamma+1}}\right)\left(\partial_{t}^{k} v_{1}^{\prime} e^{S_{0}}\right)^{\prime}-\left(1-\frac{1}{\left(\eta_{2}^{\prime}\right)^{\gamma+1}}\right)\left(\omega^{2+2 \mu} \partial_{t}^{k} v_{2}^{\prime} e^{S_{0}}\right)^{\prime}\right] \\
& -(\gamma+1) \omega^{2+2 \mu} e^{S_{0}}\left(\frac{\partial_{t}^{k} v_{1}^{\prime} \eta_{1}^{\prime \prime}}{\left(\eta_{1}^{\prime}\right)^{\gamma+2}}-\frac{\partial_{t}^{k} v_{2}^{\prime} \eta_{2}^{\prime \prime}}{\left(\eta_{2}^{\prime}\right)^{\gamma+2}}\right) .
\end{aligned}
$$

From $\eta_{i}^{\prime \prime}=\int_{0}^{t} v_{i}^{\prime \prime} d t$, using (6.2) to control $\eta_{i}^{\prime}$, by the weighted embedding inequality (2.13) and repeating elliptic estimates, we have

$$
\sup _{t \in[0, T]} \sum_{s=0}^{4}\left\|\partial_{t}^{s} \delta v\right\|_{2-s / 2}^{2} \leq C T P\left(\sup _{t \in[0, T]} \sum_{s=0}^{4}\left\|\partial_{t}^{s} \delta v\right\|_{2-s / 2}^{2}\right),
$$

which shows that $\delta v=0$.

\section{Acknowledgments}

Y. Geng's research was supported in part by the National Natural Science Foundation of China through grant 11201308 and the innovation program of Shanghai Municipal Education Commission (13ZZ136). Y. Li's research was supported in part by National Natural Science Foundation 
of China through grants 11571232 and 11831011. D. Wang's research was supported in part by the National Science Foundation under grants DMS-1312800 and DMS-1613213. R. Xu's research was supported in part by the National Natural Science Foundation of China through grant 11471087.

\section{References}

[1] Bahouri. H, Chemin. J and Danchi. R, Fourier Analysis and Nonlinear Partial differential equations. Springer Heidelburg Dordrecht London New York, 2011.

[2] Bouchut. F, Nonlinear Stability of Finite Volume Methods for Hyperbolic Conservation Laws and Well-Balanced Schemes for Sources. Frontiers in Mathematics. Birkhäuser, Basel, 2004.

[3] Chen. G.-Q, Remarks on R. J. Diperna's paper:"Convergence of the viscosity method for isentropic gas dynamics" [Comm. Math. Phys. 91 (1983) 1, 1-30]. Proc. Amer. Math. Soc. 125 (1997), 2981-2986.

[4] Coutand. D, Lindblad. H and Shkoller. S, A priori estimates for the free-boundary 3D compressible Euler equations in physical vacuum. Coтmu. Math. Phys. 296 (2010), 559-587.

[5] Coutand. D and Shkoller. S, The interaction between quasilinear elastodynamics and the Navier-Stokes equations. Arch. Ration. Mech. Anal. 179 (2006), 303-352.

[6] Coutand. D and Shkoller. S, Well-posedness of the free-surface incompressible Euler equations with or without surface tension. J. Amer. Math. Soc. 20 (2007), 829-930.

[7] Coutand. D and Shkoller. S, Well-posedness in smooth function spaces for the movingboundary 1-D compressible Euler equations in physical vacuum. Comm. Pure. Appl. Math. LXIV (2011), 328-366.

[8] Coutand. D and Shkoller. S, Well-posedness in smooth function spaces for the movingboundary 3-D compressible Euler equations in physical vacuum. Arch. Ration. Mech. Anal. 206 (2012), 515-616.

[9] Diperna. R. J, Convergence of the viscosity method for isentropic gas dynamics. Comm. Math. Phys. 91 (1983), 1-30.

[10] Greenspan. H. P and Butler. D. S, On the expansion of a gas into vacuum. J. Fluid Mech. 13 (1962), 101-119.

[11] Geng. Y and Li. Y, Local smooth solutions to the 3-dimensional isentropic relativistic Euler equations. Chin. Ann. Math. 35B(2) (2014), 301-318. 
[12] Gu. X and Lei. Z, Well-posedness of 1-D compressible Euler-Poisson equations with physical vacuum. J. Differential Equations 252 (2012), 2160-2188.

[13] Hao, C., Luo, T., A priori estimates for free boundary problem of incompressible inviscid magnetohydrodynamic flows. Arch. Rat. Mech. Anal. 212 (2014), 805-847.

[14] Hao, C, Wang, D., A priori estimates for the free boundary problem of incompressible neoHookean elastodynamics. J. Differential Equations 261 (2016), no. 1, 712-737.

[15] Huang. F, Marcati. P, and Pan. R, Convergence to the Barenblatt solution for the compressible Euler equations with damping and vacuum. Arch. Ration. Mech. Anal. 176 (2005), 1C24.

[16] Jang. J and Masmoudi. N, Well-posedness for compressible Euler equations with physical singularity. Commu. Pure Appl. Math. 62 (2009), 1327-1385.

[17] Jang. J and Masmoudi. N, Well and ill-posedness for compressible Euler equations with vacuum. J. Math. Phys. 53 (2012), 1-11.

[18] Jang. J and Masmoudi. N, Well-posedness of compressible Euler equations in a physical vacuum. Coтmu. Pure Appl. Math. 68 (2015), 61-111.

[19] Jang. J and Masmoudi. N, Vacuum in gas and fluid dynamics. Nonlinear Conservation Laws and Applications, 315-329, IMA Vol. Math. Appl. 153, Springer, New York, 2011.

[20] Jang. J, Local well-posedness of dynamics of viscous gaseous stars. Arch. Rational. Mech. Anal. 153 (2011), 315-329.

[21] Kato. T, The Cauchy problem for quasilinear symmetric hyperbolic systems. Arch. Rat. Mech. Anal. 58 (1945), 181-205.

[22] Kufner. A, Maligranda. L, Persson. L, The Hardy inequality. Vydavatelsky Servis, Plzen, 2007. About its history and some related results.

[23] Lefloch. P and Uka. S, A symmetrization of the relativistic Euler equations in sevaral spatial variables. Kinet. Relat. Modles. 2 (2009), 275-292.

[24] Lin. L, On the vacuum state for the equations of isentropic gas dynamics. J. Math. Anal. Appl. 121 (1987), 406-425.

[25] Lindblad. H, Well posedness for the motion of a compressible liquid with free surface boundary. Comm. Math. Phys. 260 (2005), 319-392.

[26] Lions. P-L, Perthame. B and Souganidis. P-E, Existence and stability of entropy solutions for the hyperbolic systems for the hyperbolic systems of isentropic gas dynamics in Eulerian and Lagrangian coordinates. Comm. Pure Appl. Math. 49 (1996), 599-638. 
Euler equations with physical vacuum

[27] Liu. T.-P, Compressible flow with damping and vacuum. Jappan. J. Indust. Appl. Math. 13 (1996), 25-32.

[28] Liu. T.-P, and J. Smoller, On the vacuum state for the isentropic gas dynamics equations. Adv. in Appl. Math. 1 (1980), 345-359.

[29] Liu. T.-P, and Yang. T, Compressible Euler equations with vacuum. J. Differential Equations. 140 (1997), 223-237.

[30] Liu. T.-P, and Yang. T, Compressible fluid with vacuum and physical singularity. Methods Appl. Anal. 7 (2000), 495-509.

[31] Luo. T and Yang. T, Interface behavior of compressible Navier-Stokes equations with vacuum. SIAM. J. Math. Anal. 31 (2000), 1175-1191.

[32] Luo. T, Xin. Z and Zeng. H, Well-posedness for the motion of physical vacuum of threedimensional compressible Euler equations with or without self-gravitation. Arch. Rational. Mech. Anal. 213 (2014), 763-831.

[33] Luo. T and Zeng. H, Global Existence of Smooth Solutions and Convergence to Barenblatt Solutions for the Physical Vacuum Free Boundary Problem of Compressible Euler Equations with Damping. Comm. Pure Appl. Math. 69(7) (2016), 1154-1396.

[34] Majda. A, Compressible fluid flow and systems of conservation laws in several space variables. Applied Mathematical Science 53, Spinger-Verlag: New York, Berlin Heidelberg, 1986.

[35] Makino. T, Ukai. S and Kawashima. S, Sur la solution à support compact de equations d'Euler compressible. Japan J. Appl. Math. 33 (1986), 249-257.

[36] Makino. T, On a local existence theorem for the evolution of gaseous stars. Pattens and Waves (eds. T. Nishida and H. Fujii), North Holland/Kinokuniya, 18 (1986), 459-479. 